\title{
INSTABILITY OF INCLINED FLUID-FILM FLOW WITH SUBSTRATE FILTRATION
}

\author{
by \\ Hom Nath Kandel \\ Masters of Mathematics Education, Tribhuvan, 2003 \\ Bachelor of Mathematics, Tribhuvan, 2001 \\ A thesis \\ presented to Ryerson University \\ in partial fulfillment of the \\ requirements for the degree of \\ Master of Science \\ in the Program of \\ Applied Mathematics \\ Toronto, Ontario, Canada, 2014 \\ (C)Hom Nath Kandel 2014
}




\section{Author's Declaration}

I hereby declare that I am the sole author of this thesis. This is a true copy of the thesis, including any required final revisions, as accepted by my examiners.

I authorize Ryerson University to lend this thesis to other institutions or individuals for the purpose of scholarly research.

I further authorize Ryerson University to reproduce this thesis by photocopying or by other means, in total or in part, at the request of other institutions or individuals for the purpose of scholarly research.

I understand that my thesis may be made electronically available to the public. 


\author{
Abstract \\ Instability of inclined fluid-film flow with substrate filtration \\ Master of Science 2014 \\ Hom Nath Kandel \\ Applied Mathematics \\ Ryerson University
}

Gravity-driven flows of thin fluid films with a free surface along a porous substrate occur in many important circumstances found in industry and natural settings. In this thesis a model for such flows is derived by coupling the Navier-Stokes equations governing the clear flow in the fluid film with Darcy's law for the filtration of fluid through the porous medium. A linear stability analysis is conducted and the effect of various parameters on the state of neutral stability is investigated. A simplified model is developed by reducing the dimensionality of the problem, which is then employed in order to determine the nonlinear effects on the stability of the equilibrium flow. 


\section{Acknowledgements}

First and foremost I offer my sincerest gratitude to my supervisor Dr. Jean-Paul Pascal who supported me throughout my thesis with his patience and knowledge. I attribute the level of my Masters degree to his encouragement and effort; without him this thesis would not have been completed or written. I am always indebted to him for his valuable time and guidance.

Besides my supervisor, I would like to thank the rest of my thesis committee: Dr. Silvana Ilie, Dr. Katrin Rohlf and Dr. Marcos Escobar for their encouragement and valuable suggestions. At the same time I would like to thank Steve Kanellis for his technical support. Also I would like to thank Ryerson University for giving me the valuable opportunity to study in the Applied Mathematics program.

Last but not the least, I would like to thank my parents, my wife Sita, my son Anupam and my daughter Alina for their support and love throughout all my studies at Ryerson University. 


\section{Dedication}

I would like to dedicate this thesis to my parents. 


\section{Contents}

1 Introduction $\quad 1$

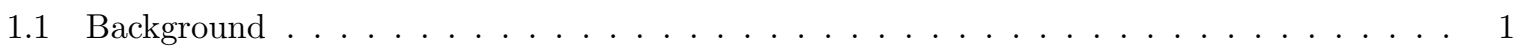

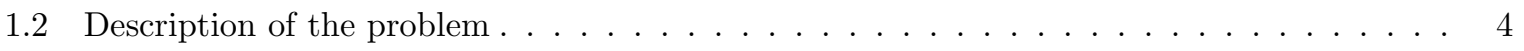

2 Governing Equations $\quad \mathbf{5}$

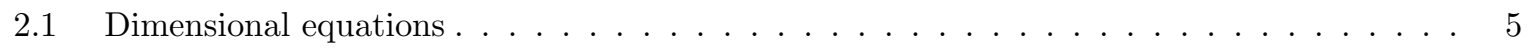

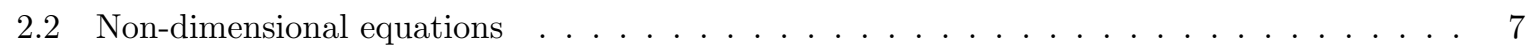

3 Linear Stability Analysis $\quad 12$

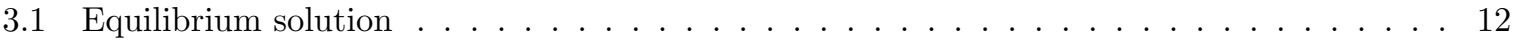

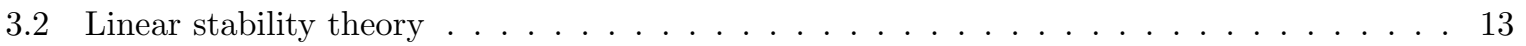

3.3 Numerical solution . . . . . . . . . . . . . . . . . . . . . . . . . . . . . 18

4 Nonlinear Effects $\quad 29$

4.1 Long-wave equations . . . . . . . . . . . . . . . . . . . . . . . 29

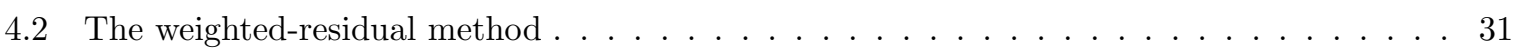

$\begin{array}{lll}5 & \text { Conclusions } & 38\end{array}$

$\begin{array}{ll}\text { References } & 40\end{array}$ 


\section{List of Figures}

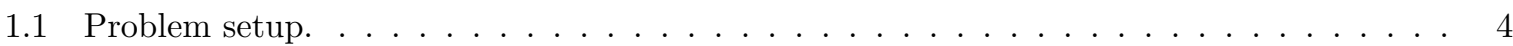

3.1 Neutral stability for different values of $D a$ with $\cot \theta=1, K a=100, \phi=0.01, \alpha_{B J}=$

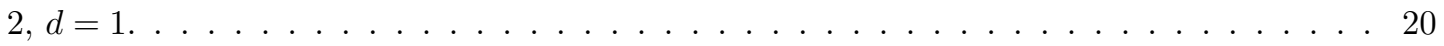

$3.2 R e_{\text {crit }}$ and $R e_{0}$ as functions of $D a$ with $\cot \theta=1, K a=100, \phi=0.01, \alpha_{B J}=2, d=1$. . . 22

3.3 Neutral stability for different values of $d$ with $\cot \theta=1, K a=100, \phi=0.01, \alpha_{B J}=$

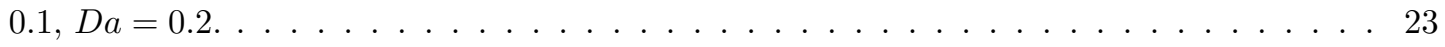

3.4 Neutral stability for different values of $K a$ with $\cot \theta=1, d=1, \phi=0.01, \alpha_{B J}=1, D a=$

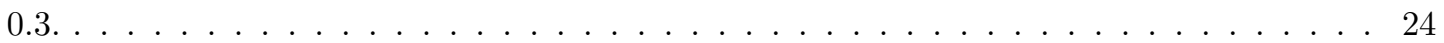

3.5 Neutral stability for different values of $\phi$ with $\cot \theta=1, d=3, K a=100, \alpha_{B J}=1, D a=$

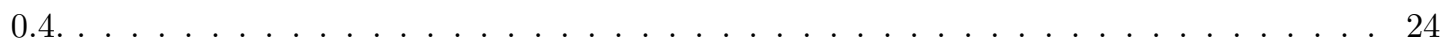

3.6 The critical Reynolds number as a function of $D a$ for different values of $\alpha_{B J}$ with $\cot \theta=$

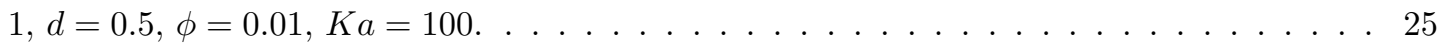

3.7 The critical Reynolds number as a function of $D a$ for different values of $\phi$ with $\cot \theta=$

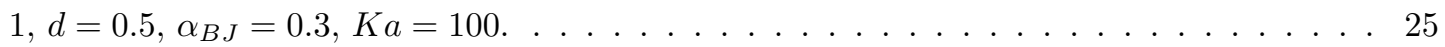

3.8 The critical Reynolds number as a function of $D a$ for different values of $d$ with $\cot \theta=$

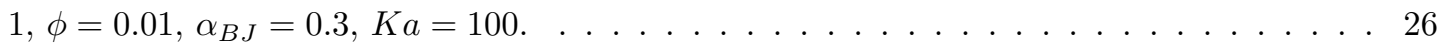

3.9 The temporal growth rate and phase speed as functions of $K$ for the impermeable bottom case $(D a=0)$, with $\cot \theta=1, K a=100$. In this case $R e_{\text {crit }}=5 / 6 \ldots \ldots \ldots \ldots$

3.10 The temporal growth rate and phase speed as functions of $K$ for $D a=0.3, \cot \theta=1, \phi=$ $0.01, d=1, \alpha_{B J}=2, K a=100$. In this case $R e_{\text {crit }} \approx 1.286 \ldots \ldots \ldots \ldots$

4.1 Neutral stability obtained by the different methods with $D a=0.3, \cot \theta=1, K a=$

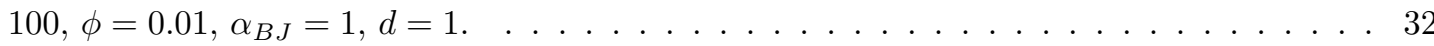

4.2 The critical Reynolds number obtained by the different methods with $\cot \theta=1, \phi=$

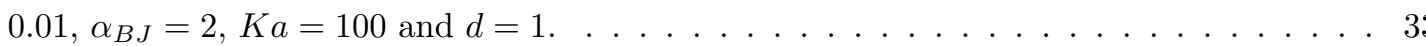

4.3 Permanent surface profiles for unstable flows with different Reynolds numbers and $D a=$ $0.3, \cot \theta=1, \phi=0.01, \alpha_{B J}=1, K a=100$ and $d=1 \ldots \ldots \ldots \ldots$ 


\section{Chapter 1}

\section{Introduction}

\section{$1.1 \quad$ Background}

Fluid films with a free surface flowing down an inclined plane are relevant in many natural and industrial settings. These flows are subject to hydrodynamic instability resulting in interfacial waves exhibiting interesting and complicated dynamical behaviour. The study of this problem has fascinated many researchers due to its conceptual simplicity, rich dynamical phenomenology and technological relevance $[1-5]$.

The pioneering experiments simulating the development of interfacial waves in film flow over an inclined plane were performed by Kapitza in 1948 [5], and additional work was then done by Kapitza and Kapitza in 1949. These investigations opened a new horizon for systematic study in this field. Kapitza and Kapitza performed experimental as well as theoretical investigations and their theory was built upon the intuitive concept of balancing work and energy supply. They identified a dimensionless number combining surface tension, kinematic viscosity and gravitation. This parameter is known as the Kapitza number and is useful in the study of the formation of interfacial waves.

More detailed theoretical investigations were later carried out by Benjamin [6] and Yih [7] who investigated the long wave instability of a falling film on an inclined plane. Resorting to linear stability analysis, Yih employed the Orr-Sommerfeld equation to determine the critical conditions for the onset of instability.

Research on inclined flow usually assumes smooth impermeable substrates, thus the no-slip and no-penetration conditions are applied. However in many realistic situations the substrate is porous or rough. Examples are found in food manufacturing, coating of photographic emulsions, the application of protective paints and so on. Consequently, the stability analysis of a falling film on an inclined porous plane is currently a developing subject. A theoretical model can be developed based on the assumption of slow filtration flow due to low permeability. This allows for the decoupling of the dynamics of the clear fluid from that of the fluid filtration through the porous medium. The result is what is referred to as a "one-sided" model which consists of flow equations for the clear fluid with the effect of substrate perme- 
ability being incorporated by means of boundary conditions at the bottom with parameters describing the permeability and porosity of the porous medium. The first such implementation was accomplished by Pascal [8] who examined the linear stability of a thin Newtonian flow down a porous incline. He employed a slip condition at the fluid-porous medium interface based on the relation proposed by Beavers and Joseph [9] under an assumption of low permeability resulting in negligible filtration velocity. In another work Pascal [10] investigated the instability of a non-Newtonian flow down a porous incline by a one-sided method. A one-dimensional model was obtained by depth integrating the long-wave equations of motion. The onset of instability was determined by means of linear theory, and a nonlinear analysis was used to study the roll waves that the instability generates.

Sadiq and Usha [11] extended the one-sided approach taken by Pascal to include the effect of surface tension on Newtonian flow. They obtained a Benny-type equation governing the thickness of the fluid layer. They carried out a weakly-nonlinear analysis and investigated how the permeability of the porous medium affects the shape, amplitude and propagation speed of the interfacial waves. Samanta et al. [12] implemented a weighted-residual method to eliminate the explicit dependence on the depth coordinate and construct a one-dimensional model for liquid film on a slippery inclined plane. They investigated the influence of slip length on the development of waves in linear and nonlinear regimes and found that a slippery substrate hastens the onset of instability, and under supercritical conditions it contributes to the increase in the amplitude of waves. Pascal and D'Alessio [13] have investigated the instability in gravity-driven flow over uneven permeable surfaces with periodic undulations. A linear stability analysis and nonlinear simulations of the evolution of perturbed steady flow were conducted to determine the critical conditions. It is shown that the destabilizing role of bottom topography is strongly dependent on the surface tension, inclination of the bottom and the wavelength of the undulations.

One-sided models have also been used to investigate non-isothermal flows along porous inclines. A study of the influence of thermocapillary effects on the flow of a film along a uniformly heated permeable incline was undertaken by Sadiq et al. [3] within the framework of long-wave linear stability analysis. An Orr-Sommerfeld system was obtained and solved asymptotically as well as numerically. The effect of heating and bottom permeability on the phase speed and the growth rate of perturbations was investigated. This problem was also considered by Ogden et al. [14], but they in addition include bottom topography in the form of periodic undulations. Furthermore, they implement a weighted-residual method to gain reduction in space dimensionality and perform nonlinear numerical simulations of the resulting equations. They found that increasing the bottom permeability monotonically destabilizes the flow. Bottom waviness stabilizes the flow if surface tension is weak but it is a destabilizing factor for strong surface tension.

In order to accurately describe cases with significant substrate permeability, a theoretical model must include the flow thorough the porous medium and couple it with that of the overlying film. This problem has received considerable attention in the study of Poiseuille flow through closed channels with porous walls. Several models have been proposed and utilised. A summary of these methods can be found, for example, in [15]. It turns out that some sort of criticism can be levelled at each of the current methodologies regarding the validity of the assumptions made, and the problem of modelling flow at a porous boundary remains open. In the absence of a clear consensus, different investigations of 
free-surface flows with a permeable substrate have resorted to various models.

Liu and Liu [16] in their investigation of flow down an inclined porous medium, have considered a coupled system consisting of the Navier-Stokes equations for the clear fluid and Darcy's law for the filtration flow in the porous medium. At the interface the Beavers-Joseph [9] condition is prescribed. In connection with linear stability theory, they obtain the corresponding Orr-Sommerfeld type equations, which are solved numerically by a Chebyshev collocation method. Conclusions are drawn regarding the significant influence of the permeability and thickness of the porous layer, as well as the Beavers-Joseph constant.

Thiele et al. [17] examined the influence of a heated porous substrate on the stability of the liquid film. Their coupled clear-filtration flow model involves the Darcy-Brinkman equation for the filtration. This equation extends Darcy's law by including an additional viscous term and thus achieving second order in spatial derivatives. Thiele et al. proceed by reducing the full system to a Benney-type equation for the evolution of the film thickness which captures the interplay between convective and thermocapillary instabilities. An important conclusion resulting from this study is that the accuracy of a one-sided model is restricted to sufficiently low permeability levels or sufficiently thin porous substrates.

Goyal et al. [18] explored the different instability modes of a pressure-driven two-layer Newtonian flow confined between a rigid wall and a porous layer. They employed the Darcy-Brinkman equation to govern the flow through the porous medium. Kumar et al. [19] also studied the instabilities of a two-layer flow over a porous substrate, but assumed a Couette flow generated by the motion of the rigid wall above the fluid layers.

Recently, Samanta et al. [4] investigated the stability of a falling film on a saturated porous inclined plane by using a continuum approach. As such, they applied a composite formulation to describe the entire clear fluid-porous medium system. This consists of a single set of equations with an assumed vertical variation of the properties of the medium. They used a weighted-residual technique and obtained equations governing the flow rate and the entire flow thickness. The results of a linear stability analysis of these equations have been compared with the results from the Orr-Sommerfeld problem. A nonlinear analysis focused on periodic waves and solitary waves was also carried out. The effect of the porous medium on the stability of the film has been found to be strongly dependent on the choice of the control parameter.

In this thesis we examine the surface instability of fluid-film flow down a porous incline, and focus on cases where the inclination is sufficiently steep for the instability to be associated with low to moderate Reynolds numbers. We model the filtration flow in the substrate by Darcy's law and couple it to the film flow by prescribing the variant of the Beavers-Joseph condition proposed by Jones [20]. In chapter 2 we set up the governing equations. In chapter 3 we carry out a linear stability analysis of the full equations. In chapter 4 we develop a simplified model based on reduction in the space dimensionality and verify its accuracy by comparing the results of the linear stability analysis with those from the full equations. We perform numerical simulations on the reduced model and investigate how nonlinear effects impact the stability of the flow. This work has been reported in [21]. 


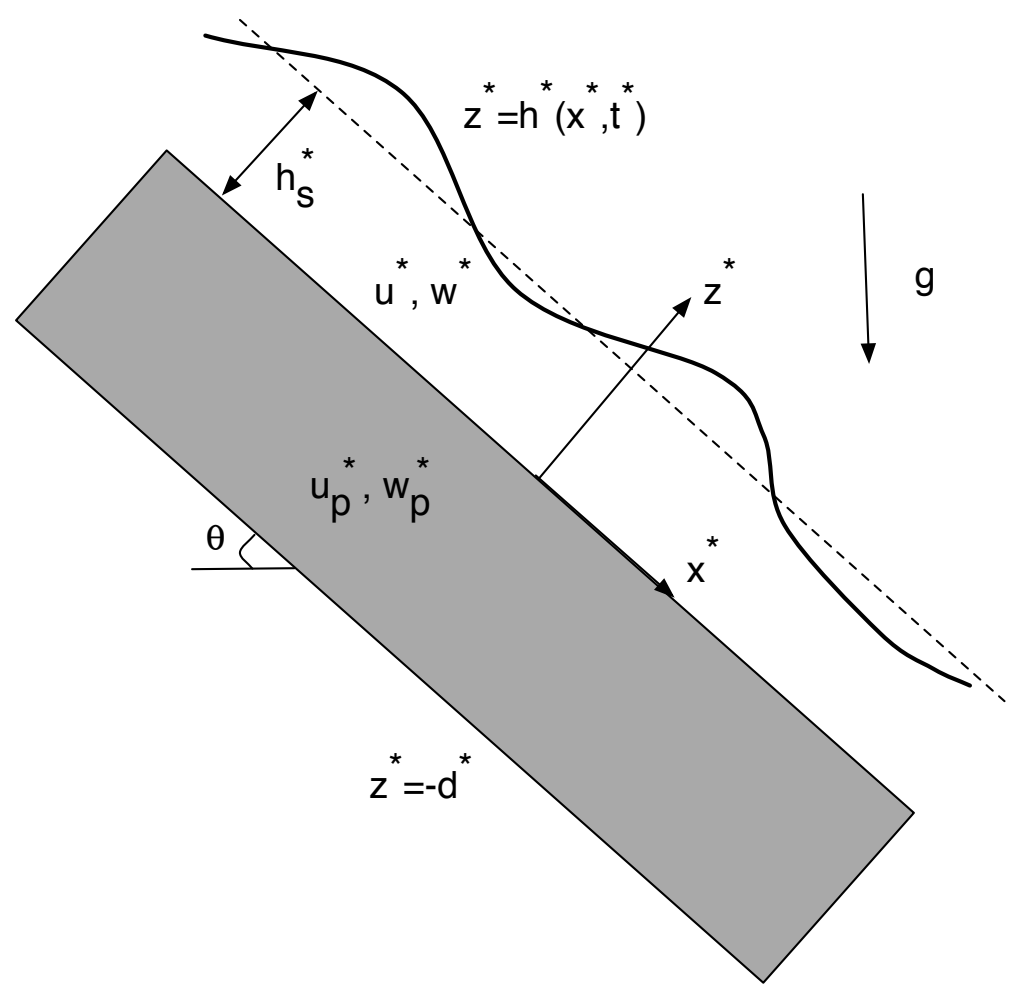

Figure 1.1: Problem setup.

\subsection{Description of the problem}

Figure 1.1 illustrates the physical model of our target study. We consider the gravity-driven twodimensional laminar flow of an incompressible Newtonian fluid down an infinitely long porous slab with planar surfaces and thickness $d^{*}$. The porous slab is saturated with the fluid and inclined at an angle $\theta$ with respect to the horizontal. An $\left(x^{*}, z^{*}\right)$ coordinate system is employed with the fluid-porous medium interface located at $z^{*}=0$. The $x^{*}$-axis points in the downhill direction and the $z^{*}$-axis points upward. The porous medium is assumed homogeneous, the flow is isothermal and the bottom surface of the porous layer is assumed to be impermeable. The thickness of the falling film is denoted by $h^{*}\left(x^{*}, t^{*}\right)$. The fluid velocity is denoted by $\mathbf{u}^{*}=\left(u^{*}, w^{*}\right)^{T}$, with the subscript $p$ marking the filtration velocity in the porous medium. We use the asterisk superscript to indicate the dimensioned variables, and then discard it for the nondimensional variables scaled as described in the next chapter. 


\section{Chapter 2}

\section{Governing Equations}

\subsection{Dimensional equations}

Equations of motion for the clear fluid flow shown in Figure 1.1 are obtained from conservation of mass which yields the continuity equation, and conservation of momentum given by the Navier-Stokes equations. The $x$-momentum equation is given by

$$
\frac{\partial u^{*}}{\partial t^{*}}+u^{*} \frac{\partial u^{*}}{\partial x^{*}}+w^{*} \frac{\partial u^{*}}{\partial z^{*}}=-\frac{1}{\rho} \frac{\partial p^{*}}{\partial x^{*}}+g \sin \theta+\frac{\mu}{\rho}\left(\frac{\partial^{2} u^{*}}{\partial x^{* 2}}+\frac{\partial^{2} u^{*}}{\partial z^{* 2}}\right)
$$

while the $z-$ momentum equation is expressed as

$$
\frac{\partial w^{*}}{\partial t^{*}}+u^{*} \frac{\partial w^{*}}{\partial x^{*}}+w^{*} \frac{\partial w^{*}}{\partial z^{*}}=-\frac{1}{\rho} \frac{\partial p^{*}}{\partial z^{*}}-g \cos \theta+\frac{\mu}{\rho}\left(\frac{\partial^{2} w^{*}}{\partial x^{* 2}}+\frac{\partial^{2} w^{*}}{\partial z^{* 2}}\right)
$$

Here $p^{*}$ is the pressure, $g$ is the acceleration due to gravity, $\rho$ is the density of the fluid and $\mu$ is the viscosity. The continuity equation can be written as

$$
\frac{\partial u^{*}}{\partial x^{*}}+\frac{\partial w^{*}}{\partial z^{*}}=0
$$

We describe momentum balance in the filtration flow through the porous medium by Darcy's law. This model is based on the intrinsic assumption of a proportionality between flow rate and applied pressure. For the inclined filtration flow referred to in Figure 1.1 Darcy's law is expressed as

$$
\begin{gathered}
\frac{\rho}{\phi} \frac{\partial u_{p}^{*}}{\partial t^{*}}=-\frac{\partial p_{p}^{*}}{\partial x^{*}}-\frac{\mu}{\kappa} u_{p}^{*}+g \rho \sin \theta \\
\frac{\rho}{\phi} \frac{\partial w_{p}^{*}}{\partial t^{*}}=-\frac{\partial p_{p}^{*}}{\partial z^{*}}-\frac{\mu}{\kappa} w_{p}^{*}-g \rho \cos \theta,
\end{gathered}
$$


where $p_{p^{*}}^{*}$ is the intrinsic volume averaged pressure, $\phi$ is the porosity of the porous medium and $\kappa$ denotes its permeability.

The continuity equation for the filtration flow is given by

$$
\frac{\partial u_{p}^{*}}{\partial x^{*}}+\frac{\partial w_{p}^{*}}{\partial z^{*}}=0
$$

Conditions at the free surface of the fluid film are obtained from continuity of force and conservation of mass. Now, the total stress tensor associated with the flow is given by

$\overrightarrow{\vec{s}}=-p^{*} \stackrel{\vec{I}}{\vec{\tau}}+\overrightarrow{\vec{\tau}}$, where

$$
\vec{\tau}=\mu\left[\begin{array}{cc}
2 \frac{\partial u^{*}}{\partial x^{*}} & \frac{\partial u^{*}}{\partial z^{*}}+\frac{\partial w^{*}}{\partial x^{*}} \\
\frac{\partial u^{*}}{\partial z^{*}}+\frac{\partial w^{*}}{\partial x^{*}} & 2 \frac{\partial w^{*}}{\partial z^{*}}
\end{array}\right] \text { and } \vec{I}=\left[\begin{array}{ll}
1 & 0 \\
0 & 1
\end{array}\right]
$$

The stress vector acting on surface $z^{*}=h^{*}\left(x^{*}, t^{*}\right)$ is then $\vec{s} \hat{n}$, where

$$
\widehat{n}=\frac{1}{\sqrt{1+\left(\frac{\partial h^{*}}{\partial x^{*}}\right)^{2}}}\left[\begin{array}{c}
-\frac{\partial h^{*}}{\partial x^{*}} \\
1
\end{array}\right]
$$

is the unit normal vector. We assume the ambient gas above the clear fluid film to be dynamically passive. Consequently, the normal force exerted by the flow on the surface is balanced by surface tension with no contribution from the ambient gas. This is expressed mathematically as $(\vec{s} \widehat{n}) \cdot \widehat{n}=\gamma \mathcal{C}$, where $\gamma$ is the surface tension and $\mathcal{C}$ measures the mean curvature of the surface. Expanding this condition we obtain

$$
\begin{array}{r}
-p^{*}+\frac{2 \mu}{1+\left(\frac{\partial h^{*}}{\partial x^{*}}\right)^{2}}\left[\left(\frac{\partial h^{*}}{\partial x^{*}}\right)^{2} \frac{\partial u^{*}}{\partial x^{*}}+\frac{\partial w^{*}}{\partial z^{*}}-\frac{\partial h^{*}}{\partial x^{*}}\left(\frac{\partial u^{*}}{\partial z^{*}}+\frac{\partial w^{*}}{\partial x^{*}}\right)\right] \\
=\frac{\gamma \frac{\partial^{2} h^{*}}{\partial x^{* 2}}}{\left(1+\left(\frac{\partial h^{*}}{\partial x^{*}}\right)^{2}\right)^{\frac{3}{2}}} \text { at } z^{*}=h^{*}
\end{array}
$$

If we further assume that the surface tension is constant, then the tangential component of the force exerted by the flow on the surface must equal zero, and we have $(\vec{s} \widehat{n}) \cdot \widehat{t}=0$, where

$$
\widehat{t}=\frac{1}{\sqrt{1+\left(\frac{\partial h^{*}}{\partial x^{*}}\right)^{2}}}\left[\begin{array}{c}
1 \\
\frac{\partial h^{*}}{\partial x^{*}}
\end{array}\right]
$$

is the unit tangent vector to the surface. It then follows that

$$
\frac{\mu}{1+\left(\frac{\partial h^{*}}{\partial x^{*}}\right)^{2}}\left[-4 \frac{\partial h^{*}}{\partial x^{*}} \frac{\partial u^{*}}{\partial x^{*}}+\left(1-\left(\frac{\partial h^{*}}{\partial x^{*}}\right)^{2}\right)\left(\frac{\partial u^{*}}{\partial z^{*}}+\frac{\partial w^{*}}{\partial x^{*}}\right)\right]=0 \quad \text { at } \quad z^{*}=h^{*} .
$$

To obtain a kinematic condition for the surface, we assume that evaporation is negligible. Conser- 
vation of mass then dictates that fluid particles on the surface of the fluid layer stay on the surface as the layer flows. Consequently, the vertical component of the fluid velocity at the surface must equal the material derivative of the height of the surface. This condition is expressed as

$$
w^{*}=\frac{D h^{*}}{D t^{*}} \equiv \frac{\partial h^{*}}{\partial t^{*}}+\frac{\partial h^{*}}{\partial x^{*}} u^{*} \quad \text { at } \quad z^{*}=h^{*} .
$$

At the fluid-porous medium interface, located at $z^{*}=0$, we assume continuity of normal stress which is expressed as

$$
-p^{*}+2 \mu \frac{\partial w^{*}}{\partial z^{*}}=-p_{p}^{*}+2 \mu \frac{\partial w_{p}^{*}}{\partial z^{*}} \quad \text { at } \quad z^{*}=0 .
$$

Here we also impose continuity of vertical velocity

$$
w^{*}=w_{p}^{*} \quad \text { at } \quad z^{*}=0 .
$$

The slip-velocity condition at a fluid-porous medium interface originally proposed by Beavers and Joseph [9] stipulates a proportionality between velocity shear and the difference between the clear fluid velocity and filtration velocity at the interface. For our problem we will employ the modified version proposed by Jones [20] which replaces the velocity shear by shear stress and is expressed as

$$
\frac{\partial u^{*}}{\partial z^{*}}+\frac{\partial w^{*}}{\partial x^{*}}=\frac{\alpha_{B J}}{\sqrt{\kappa}}\left(u^{*}-u_{p}^{*}\right) \quad \text { at } \quad z^{*}=0
$$

where $\alpha_{B J}$ is the Beaver-Joseph parameter, an empirical quantity dependent on the pore-space geometry of the porous medium. Experimental results [9] indicate that this parameter should be assigned values between 0.1 and 4 .

At the bottom of the porous layer we assume an impermeable surface and consequently the appropriate boundary condition is

$$
w_{p}^{*}=0 \quad \text { at } \quad z^{*}=-d^{*},
$$

where $d^{*}$ is the thickness of porous medium.

\subsection{Non-dimensional equations}

The steady and laterally-uniform solution to our model can be expressed as a relation between the discharge and thickness of the clear film, involving the properties of the fluid and the inclination and permeability of the porous substrate. The possibilities therefore are to regard the discharge as the prescribed quantity, or to consider the thickness as being given. In the latter case, the prescribed film thickness can be used as the vertical length scale. However, in this study we choose to assume that a discharge is prescribed. In this case, the resulting film thickness of the steady and uniform flow depends on the permeability of the substrate, and as a result it would not be an appropriate choice for a length scale. This is due to the fact that it would then be difficult to determine the actual effect of 
the permeability on the results. For the vertical length scale we instead employ

$$
H=\left(\frac{3 \mu Q}{\rho g \sin \theta}\right)^{1 / 3}
$$

where $Q$ is the prescribed discharge. We point out that $H$ coincides with the Nusselt thickness of flow over an impermeable surface. For the cases considered in our study, this quantity is of the same order of magnitude as the thickness of the uniform film flowing over a permeable substrate. Consequently, $H$ is a characteristic film thickness for our problem. Another possibility for the vertical length scale is the thickness of the porous slab. However, this length may not be of the same order of magnitude as the clear film thickness and consequently, when scaled this variable may be equal to large values which would complicate the analysis. The velocity scale will consequently be $U=Q / H$, with the pressure being scale by $\rho U^{2}$. In the horizontal direction we employ a different length scale and use $\delta$ to denote the aspect ratio of vertical to horizontal length scales. Dividing by the velocity scale gives us the time scale $H /(U \delta)$. The use of different length scales will be relevant when we make approximations based on a long-wave assumption.

We employ the scaling described above to non-dimensionalize the equations governing the film flow and use the notation without the asterisk to denote the scaled variables. As such, the continuity equation becomes

$$
\frac{\partial(U u)}{\partial\left(\frac{H}{\delta} x\right)}+\frac{\partial(\delta U w)}{\partial(H z)}=0
$$

which, when simplified becomes

$$
\frac{\partial u}{\partial x}+\frac{\partial w}{\partial z}=0
$$

The momentum equations in the $x$ and $z$ directions transform into respectively

$$
\frac{\partial(U u)}{\partial\left(\frac{H}{U \delta} t\right)}+(U u) \frac{\partial(U u)}{\partial\left(\frac{H}{\delta} x\right)}+(\delta U w) \frac{\partial(U u)}{\partial(H z)}=-\frac{1}{\rho} \frac{\partial\left(\rho U^{2} p\right)}{\partial\left(\frac{H}{\delta} x\right)}+g \sin \theta+\frac{\mu}{\rho}\left(\frac{\partial^{2}(U u)}{\partial\left(\frac{H}{\delta} x\right)^{2}}+\frac{\partial^{2}(U u)}{\partial(H z)^{2}}\right)
$$

and

$$
\frac{\partial(\delta U w)}{\partial\left(\frac{H}{U \delta} t\right)}+(U u) \frac{\partial(\delta U w)}{\partial\left(\frac{H}{\delta} x\right)}+(\delta U w) \frac{\partial(\delta U w)}{\partial(H z)}=-\frac{1}{\rho} \frac{\partial\left(\rho U^{2} p\right)}{\partial(H z)}-g \cos \theta+\frac{\mu}{\rho}\left(\frac{\partial^{2}(\delta U w)}{\partial\left(\frac{H}{\delta} x\right)^{2}}+\frac{\partial^{2}(\delta U w)}{\partial(H z)^{2}}\right) .
$$

These simplify to become

$$
\delta R e\left(\frac{\partial u}{\partial t}+u \frac{\partial u}{\partial x}+w \frac{\partial u}{\partial z}\right)=-\delta R e \frac{\partial p}{\partial x}+3+\delta^{2} \frac{\partial^{2} u}{\partial x^{2}}+\frac{\partial^{2} u}{\partial z^{2}}
$$




$$
\delta^{2} R e\left(\frac{\partial w}{\partial t}+u \frac{\partial w}{\partial x}+w \frac{\partial w}{\partial z}\right)=-\operatorname{Re} \frac{\partial p}{\partial z}-3 \cot \theta+\delta^{3} \frac{\partial^{2} w}{\partial x^{2}}+\delta \frac{\partial^{2} w}{\partial z^{2}}
$$

where $R e$ is the Reynolds number defined as $R e=\frac{\rho Q}{\mu}$.

In the filtration flow through the porous substrate we scale the filtration velocity by $U_{p}=\frac{\kappa U}{H^{2}}$ and the pressure by $P_{p}=\frac{\rho U^{2}}{R e \delta}$. Scaling the conservation of mass equation for the filtration flow gives

$$
\frac{\partial\left(U_{p} u_{p}\right)}{\partial\left(\frac{H}{\delta} x\right)}+\frac{\partial\left(\delta U_{p} w_{p}\right)}{\partial(H z)}=0
$$

which reduces to

$$
\frac{\partial u_{p}}{\partial x}+\frac{\partial w_{p}}{\partial z}=0
$$

Applying the scaling to the $x$-momentum equation for the filtration flow gives

$$
\frac{\rho}{\phi} \frac{\partial\left(U_{p} u_{p}\right)}{\partial\left(\frac{H}{U \delta} t\right)}=-\frac{\partial\left(P_{p} p_{p}\right)}{\partial\left(\frac{H}{\delta} x\right)}-\frac{\mu}{\kappa}\left(U_{p} u_{p}\right)+g \rho \sin \theta
$$

which simplifies to

$$
\frac{D a^{2} R e \delta}{\phi} \frac{\partial u_{p}}{\partial t}=-\frac{\partial p_{p}}{\partial x}-u_{p}+3
$$

where $D a=\sqrt{\kappa} / H$ is the Darcy number which measures the permeability of the porous medium to fluid filtration. And the scaled $z$-momentum equation in the porous substrate is given by

$$
\frac{\rho}{\phi} \frac{\partial\left(\delta U_{p} w_{p}\right)}{\partial\left(\frac{H}{U \delta} t\right)}=-\frac{\partial\left(P_{p} p_{p}\right)}{\partial(H z)}-\frac{\mu}{\kappa}\left(\delta U_{p} w_{p}\right)-g \rho \cos \theta
$$

from which we obtain

$$
\frac{D a^{2} R e \delta^{3}}{\phi} \frac{\partial w_{p}}{\partial t}=-\frac{\partial p_{p}}{\partial z}-\delta^{2} w_{p}-3 \delta \cot \theta
$$

Scaling the condition of continuity of normal force at the surface we obtain

$$
\begin{aligned}
\rho U^{2} p & -\frac{2 \mu}{\left(1+\left(\frac{\partial(H h)}{\partial\left(\frac{H}{\delta} x\right)}\right)^{2}\right)}\left[\left(\frac{\partial(H h)}{\partial\left(\frac{H}{\delta} x\right)}\right)^{2} \frac{\partial(U u)}{\partial\left(\frac{H}{\delta} x\right)}+\frac{\partial(\delta U w)}{\partial(H z)}-\frac{\partial(H h)}{\partial\left(\frac{H}{\delta} x\right)}\left(\frac{\partial(U u)}{\partial(H z)}+\frac{\partial(\delta U w)}{\partial\left(\frac{H}{\delta} x\right)}\right)\right] \\
& +\frac{\gamma \frac{\partial^{2}(H h)}{\partial\left(\frac{H}{\delta} x\right)^{2}}}{\left(1+\left(\frac{\partial(H h)}{\partial\left(\frac{H}{\delta} x\right)}\right)^{2}\right)^{\frac{3}{2}}}=0 \quad \text { at } \quad z=h(x, t) .
\end{aligned}
$$


Simplified, this equation can be written as

$$
\begin{gathered}
p=\quad \frac{2 \delta}{\operatorname{Re}\left[1+\delta^{2}\left(\frac{\partial h}{\partial x}\right)^{2}\right]}\left[\delta^{2}\left(\frac{\partial h}{\partial x}\right)^{2} \frac{\partial u}{\partial x}+\frac{\partial w}{\partial z}-\frac{\partial h}{\partial x} \frac{\partial u}{\partial z}-\delta^{2} \frac{\partial h}{\partial x} \frac{\partial w}{\partial x}\right] \\
-\frac{\delta^{2} W e}{\left[1+\delta^{2}\left(\frac{\partial h}{\partial x}\right)^{2}\right]^{3 / 2}} \frac{\partial^{2} h}{\partial x^{2}} \text { at } z=h(x, t),
\end{gathered}
$$

where $W e=\left(\frac{3}{R e^{5} \sin \theta}\right)^{1 / 3} K a$ and $K a$ denotes the Kapitza number which is the scaled surface tension given by $K a=\gamma /\left(g^{1 / 3} \rho \nu^{4 / 3}\right)$, with $\gamma$ being the surface tension and $\nu$ the kinematic viscosity, which equals $\mu / \rho$.

Scaling the tangential component of the continuity of force condition at the surface yields

$\frac{\mu}{1+\left(\frac{\partial(H h)}{\partial\left(\frac{H}{\delta} x\right)}\right)^{2}}\left[-4 \frac{\partial(H h)}{\partial\left(\frac{H}{\delta} x\right)} \frac{\partial(U u)}{\partial\left(\frac{H}{\delta} x\right)}+\left(1-\left(\frac{\partial(H h)}{\partial\left(\frac{H}{\delta} x\right)}\right)^{2}\right)\left(\frac{\partial(U u)}{\partial(H z)}+\frac{\partial(\delta U w)}{\partial\left(\frac{H}{\delta} x\right)}\right)\right]=0 \quad$ at $\quad z=h(x, t)$,

which then gives

$$
\left[1-\delta^{2}\left(\frac{\partial h}{\partial x}\right)^{2}\right]\left(\frac{\partial u}{\partial z}+\delta^{2} \frac{\partial w}{\partial x}\right)-4 \delta^{2} \frac{\partial h}{\partial x} \frac{\partial u}{\partial x}=0 \quad \text { at } \quad z=h(x, t) .
$$

The kinematic condition becomes

$$
\delta U w=\frac{\partial(H h)}{\partial\left(\frac{H}{U \delta} t\right)}+\frac{\partial(H h)}{\partial\left(\frac{H}{\delta} x\right)}(U u) \quad \text { at } \quad z=h .
$$

This, upon simplifying, gives

$$
w=\frac{\partial h}{\partial t}+u \frac{\partial h}{\partial x} \quad \text { at } \quad z=h
$$

Scaling the continuity of normal stress condition at the fluid-porous interface leads to

$$
-\rho U^{2} p+2 \mu \frac{\partial(\delta U w)}{\partial(H z)}=-\frac{\rho U^{2}}{\delta R e} p_{p}+2 \mu \frac{\partial\left(\delta U_{p} w_{p}\right)}{\partial(H z)} \quad \text { at } \quad z=0
$$

and simplifying results in

$$
\delta R e p-2 \delta^{2} \frac{\partial w}{\partial z}=p_{p}-2 D a^{2} \delta^{2} \frac{\partial w_{p}}{\partial z} .
$$

The scaled continuity of vertical velocity condition is

$$
w=D a^{2} w_{p} \quad \text { at } \quad z=0 .
$$


And the shear stress condition at $z=0$ is

$$
\frac{\partial(U u)}{\partial(H z)}+\frac{\partial(\delta U w)}{\partial\left(\frac{H}{\delta} x\right)}=\frac{\alpha_{B J}}{\sqrt{\kappa}}\left(U u-U_{p} u_{p}\right)
$$

which gives

$$
\frac{\partial u}{\partial z}+\delta^{2} \frac{\partial w}{\partial x}=\frac{\alpha_{B J}}{D a}\left(u-D a^{2} u_{p}\right)
$$

At the bottom of the porous substrate, i.e. at $z=-d$,

$$
w_{p}=0
$$

where $d=d^{*} / H$ and is thus the ratio of the thickness of the porous medium and a characteristic thickness of the clear fluid film.

As it will become apparent in the later chapters, qualitative changes occur in the results of our analysis for sufficiently large values of the Darcy number. It is thus important to specify, at least in terms of magnitude, the upper limit of the range of realistic values of this parameter. Now, as stated above, $D a=\sqrt{\kappa} / H$, so we first determine how small the characteristic film thickness, $H$, can be. It turns out that inertial instability, which is the focus of our investigation, can be important even in very thin film flows, with the thickness being significantly smaller than a millimetre [5]. Next, regarding large permeability values, we point out that fibrous materials can display high levels of hydrodynamic conductivity. The observations from several experimental investigations involving fibrous porous media, as tabulated by Jackson and James [22], indicate that such materials can have permeabilities of order of magnitude as large as that of $1 \mathrm{~mm}^{2}$. Therefore, with $H=1 \mathrm{~mm}$ for example, realistic Darcy numbers extend up to values of the same order of magnitude as 1 . 


\section{Chapter 3}

\section{Linear Stability Analysis}

The equations of motion (2.14) - (2.19) together with the boundary conditions (2.20) - (2.26) provide a mathematical model for film flow with substrate filtration. The model admits a simple equilibrium solution which corresponds to the steady flow of a fluid film with uniform thickness. The question is, however, whether this flow can persist or if it is hydrodynamically unstable. In this chapter we investigate the stability of the steady flow by means of a linear stability analysis. This theory determines the temporal growth rate of infinitesimal perturbations imposed on the flow and thus predicts under what conditions these are amplified in time.

\subsection{Equilibrium solution}

If we set the $t$ and $x$ derivatives to zero in our governing equations, (2.14) - (2.26), it becomes evident that the problem is satisfied by $w \equiv 0, w_{p} \equiv 0, u_{p} \equiv 3$, and the remaining dependent variables satisfying the differential equations

$$
\frac{\partial^{2} u}{\partial z^{2}}=-3, \quad \frac{\partial p}{\partial z}=-3 \frac{\cot \theta}{R e} \quad \text { and } \quad \frac{\partial p_{p}}{\partial z}=-3 \delta \cot \theta
$$

with conditions at $z=h_{s}$ given by

$$
\frac{\partial u}{\partial z}=0 \quad \text { and } \quad p=0
$$

where $h_{s}$ is a constant to be determined and corresponds to the uniform thickness of the equilibrium flow. At $z=0$ the conditions reduce to

$$
\frac{\partial u}{\partial z}=\frac{\alpha_{B J}}{D a}\left(u-3 D a^{2}\right) \quad \text { and } \quad \delta \operatorname{Re} p=p_{p} .
$$


Solving this problem we obtain

$$
\begin{gathered}
p=p_{s}(z) \equiv 3 \frac{\cot \theta}{R e}\left(h_{s}-z\right) \\
p_{p}=p_{p_{s}}(z) \equiv 3 \delta \cot \theta\left(h_{s}-z\right)
\end{gathered}
$$

and

$$
u=u_{s}(z) \equiv 3 h_{s} z-\frac{3}{2} z^{2}+3 \frac{D a}{\alpha_{B J}} h_{s}+3 D a^{2} .
$$

To determine the value of $h_{s}$ we use the fact that our scaling is based on the prescribed flow rate. This requires the scaled flow rate to be unity, i.e.

$$
\int_{0}^{h_{s}} u_{s}(z) d z=1
$$

This leads to

$$
h_{s}^{3}+3 \frac{D a}{\alpha_{B J}} h_{s}^{2}+3 D a^{2} h_{s}-1=0,
$$

which can be solved for $h_{s}$ to give

$$
h_{s}=\frac{1}{2 r \alpha_{B J}}\left(r^{2}-2 r D a+4 D a^{2}-4 D a^{2} \alpha_{B J}^{2}\right),
$$

where

$r=\left(12 D a^{3} \alpha_{B J}{ }^{2}+4 \alpha_{B J}^{3}-8 D a^{3}+4 \alpha_{B J}^{3 / 2} \sqrt{4 D a^{6} \alpha_{B J^{3}}-3 D a^{6} \alpha_{B J}+6 D a^{3} \alpha_{B J}{ }^{2}+\alpha_{B J}{ }^{3}-4 D a^{3}}\right)^{1 / 3}$.

In the next section we study the stability of the equilibrium solution given by $w \equiv 0, w_{p} \equiv 0, u_{p} \equiv 3$, $u=u_{s}(z), p=p_{s}(z), p_{p}=p_{p_{s}}(z)$ and $h=h_{s}$.

\subsection{Linear stability theory}

We consider the perturbed equilibrium solution expressed as

$$
\begin{gathered}
h=h_{s}+\eta(x, t), \quad u=u_{s}(z)+\tilde{u}(x, z, t), \quad w=\tilde{w}(x, z, t), \quad p=p_{s}(z)+\tilde{p}(x, z, t), \\
u_{p}=3+\tilde{u_{p}}(x, z, t), \quad w_{p}=\tilde{w}_{p}(x, z, t) \quad \text { and } \quad p_{p}=p_{p s}(z)+\tilde{p_{p}}(x, z, t),
\end{gathered}
$$

where $\eta$ and the quantities with the tildes are the added infinitesimal perturbations. Introducing this perturbed state into equations (2.14) - (2.26) and linearizing with respect to the infinitesimal disturbances, 
we obtain

$$
\begin{gathered}
\frac{\partial \tilde{u}}{\partial x}+\frac{\partial \tilde{w}}{\partial z}=0 \\
\delta R e\left(\frac{\partial \tilde{u}}{\partial t}+u_{s} \frac{\partial \tilde{u}}{\partial x}+\tilde{w} \frac{\partial u_{s}}{\partial z}\right)=-\delta R e \frac{\partial \tilde{p}}{\partial x}+\delta^{2} \frac{\partial^{2} \tilde{u}}{\partial x^{2}}+\frac{\partial^{2} \tilde{u}}{\partial z^{2}} \\
\delta^{2} \operatorname{Re}\left(\frac{\partial \tilde{w}}{\partial t}+u_{s} \frac{\partial \tilde{w}}{\partial x}\right)=-\operatorname{Re} \frac{\partial \tilde{p}}{\partial z}+\delta^{3} \frac{\partial^{2} \tilde{w}}{\partial x^{2}}+\delta \frac{\partial^{2} \tilde{w}}{\partial z^{2}} \\
\frac{\partial \tilde{u_{p}}}{\partial x}+\frac{\partial \tilde{w_{p}}}{\partial z}=0 \\
\frac{D a^{2} R e \delta}{\phi} \frac{\partial \tilde{u_{p}}}{\partial t}=-\frac{\partial \tilde{p_{p}}}{\partial x}-\tilde{u_{p}}
\end{gathered}
$$

and

$$
\frac{D a^{2} \operatorname{Re} \delta^{3}}{\phi} \frac{\partial \tilde{w}_{p}}{\partial t}=-\frac{\partial \tilde{p_{p}}}{\partial z}-\delta^{2} \tilde{w}_{p}
$$

The boundary conditions for these perturbation equations, evaluated at $z=h_{s}$, are

$$
\begin{gathered}
\tilde{p}-\frac{3}{R e} \cot \theta \eta-\frac{2 \delta}{R e} \frac{\partial \tilde{w}}{\partial z}+\delta^{2} W e \frac{\partial^{2} \eta}{\partial x^{2}}=0 \\
-3 \eta+\frac{\partial \tilde{u}}{\partial z}+\delta^{2} \frac{\partial \tilde{w}}{\partial x}=0
\end{gathered}
$$

and

$$
\tilde{w}=\frac{\partial \eta}{\partial t}+u_{s} \frac{\partial \eta}{\partial x} .
$$

At $z=0$ the conditions are

$$
\begin{gathered}
\delta \operatorname{Re} \tilde{p}-2 \delta^{2} \frac{\partial \tilde{w}}{\partial z}=\tilde{p_{p}}-2 D a^{2} \delta^{2} \frac{\partial \tilde{\tilde{w}_{p}}}{\partial z} \\
\frac{\partial \tilde{u}}{\partial z}+\delta^{2} \frac{\partial \tilde{w}}{\partial x}=\frac{\alpha_{B J}}{D a}\left(\tilde{u}-D a^{2} \tilde{u_{p}}\right)
\end{gathered}
$$


and

$$
\tilde{w}=D a^{2} \tilde{w}_{p}
$$

And finally, at $z=-d$ the boundary condition is

$$
\tilde{w}_{p}=0
$$

Into the linearized perturbation equations we introduce normal modes according to

$$
\left(\tilde{u}, \tilde{u_{p}}, \tilde{w}, \tilde{w_{p}}, \tilde{p}, \tilde{p_{p}}, \eta\right)=\left(\hat{u}(z), \hat{u_{p}}(z), \hat{w}(z), \hat{w}_{p}(z), \hat{p}(z), \hat{p_{p}}(z), \hat{\eta}\right) e^{i k(x-c t)},
$$

where $k$ is a real positive quantity representing the wavenumber of the perturbation and $c$ is a complex quantity whose real part is the phase speed of the perturbation while the imaginary part multiplied by $k$ is the temporal growth rate. The equations governing the amplitudes of the normal modes are

$$
\begin{gathered}
D \hat{w}+i k \hat{u}=0 \\
\delta \operatorname{Re}\left[i k\left(u_{s}-c\right) \hat{u}+D u_{s} \hat{w}\right]=-i k \delta \operatorname{Re} \hat{p}+D^{2} \hat{u}-k^{2} \delta^{2} \hat{u} \\
i k \delta^{2} \operatorname{Re}\left(u_{s}-c\right) \hat{w}=-\operatorname{Re} \hat{D}+\delta D^{2} \hat{w}-k^{2} \delta^{3} \hat{w} \\
D \hat{w}_{p}+i k \hat{u_{p}}=0 \\
\left(i k c \frac{D a^{2} \operatorname{Re} \delta}{\phi}-1\right) \hat{u_{p}}=i k \hat{p_{p}} \\
\left(i k c \frac{D a^{2} \operatorname{Re} \delta^{3}}{\phi}-\delta^{2} \hat{w}_{p}\right)=D \hat{p_{p}},
\end{gathered}
$$

where $D$ is a differential operator denoting differentiation with respect to $z$. Accompanying these equations we have boundary conditions evaluated at $z=h_{s}$ expressed as

$$
\begin{gathered}
\hat{p}-\frac{3}{R e} \cot \theta \hat{\eta}-\frac{2 \delta}{R e} D \hat{w}-k^{2} \delta^{2} W e \hat{\eta}=0 \\
-3 \hat{\eta}+D \hat{u}+i k \delta^{2} \hat{w}=0 \\
\hat{w}=i k \hat{\eta}\left(u_{s}-c\right) .
\end{gathered}
$$


The boundary conditions at $z=0$ are

$$
\begin{aligned}
& \delta R e \hat{p}-2 \delta^{2} D \hat{w}=\hat{p_{p}}-2 D a^{2} \delta^{2} D \hat{w_{p}} \\
& D \hat{u_{p}}+\delta^{2} i k \hat{w}_{p}=\frac{\alpha_{B J}}{D a}\left(\hat{u}-D a^{2} \hat{u_{p}}\right)
\end{aligned}
$$

and

$$
\hat{w}=D a^{2} \hat{w}_{p}
$$

At $z=-d$ we have

$$
\hat{w}_{p}=0 \text {. }
$$

Eliminating $\hat{p}$ from equations (3.19) and (3.20) we obtain

$$
\begin{aligned}
& i k \delta \operatorname{Re} D u_{s} \hat{u}+\left[i k \delta \operatorname{Re}\left(u_{s}-c\right)+k^{2} \delta^{2}\right] D \hat{u}+\operatorname{Re} \delta D u_{s} D \hat{w}+\operatorname{Re} \delta D^{2} u_{s} \hat{w}-D^{3} \hat{u} \\
& =-k^{2} \delta^{3} \operatorname{Re}\left(u_{s}-c\right) \hat{w}-i k \delta^{2} D^{2} \hat{w}+i k^{3} \delta^{4} \hat{w}
\end{aligned}
$$

Similarly, eliminating $\hat{p_{p}}$ from equations (3.22) and (3.23) yields

$$
\left(1-i k c \frac{\operatorname{Re} \delta}{\phi}\right) D \hat{u_{p}}=i k\left(i k c R e \frac{\delta^{3}}{\phi}+\delta^{2}\right) \hat{w}_{p} .
$$

Eliminating $\hat{p}$ and $\hat{p_{p}}$ from the boundary conditions gives

$$
\begin{array}{r}
D^{2} \hat{u}-\delta^{2} k^{2} \hat{u}-3 i \delta k \cot \theta \hat{\eta}-2 i \delta^{2} k D \hat{w}-i \delta^{3} k^{3} \operatorname{ReWe\hat {\eta }-} \\
\operatorname{Re} \delta\left[i k\left(u_{s}-c\right) \hat{u}-D u_{s} \hat{w}\right]=0 \quad \text { at } \quad z=h_{s} \\
D^{2} \hat{u}-\delta k^{2} \hat{u}-\operatorname{Re} \delta\left[i k\left(u_{s}-c\right) \hat{u}+D u_{s} \hat{w}\right]-2 i \delta^{2} k D \hat{w} \\
=\frac{\operatorname{Re} \delta}{\phi} i c k \hat{u_{p}}-\hat{u_{p}}-2 i \delta^{2} k D a^{2} D \hat{w} \quad \text { at } \quad z=0 .
\end{array}
$$

Equations (3.18) and (3.21) can be eliminated by expressing the velocity disturbances in terms of the stream function as

$$
\tilde{u}=\frac{\partial \psi}{\partial z}, \tilde{w}=-\frac{\partial \psi}{\partial x}, \tilde{u_{p}}=\frac{\partial \psi_{p}}{\partial z}, \tilde{w_{p}}=-\frac{\partial \psi_{p}}{\partial x} .
$$

Introducing the normal modes for the stream functions expressed as

$$
\left(\psi, \psi_{p}\right)=\left(\Psi(z), \Psi_{p}(z)\right) e^{i k(x-c t)},
$$


leads to the following Orr-Sommerfeld type equations

$$
\begin{gathered}
D^{4} \Psi-\left[i K \operatorname{Re}\left(u_{s}-c\right)+2 K^{2}\right] D^{2} \Psi+\left[i K^{3} \operatorname{Re}\left(u_{s}-c\right)+i K \operatorname{Re} D^{2} u_{s}+K^{4}\right] \Psi=0, \\
D^{2} \Psi_{p}-K^{2} \Psi_{p}=0,
\end{gathered}
$$

where $K=k \delta$. Boundary conditions for these equations evaluated at $z=h_{s}$ are

$$
\begin{gathered}
D^{3} \Psi-\left[i K R e\left(u_{s}-c\right)+3 K^{2}\right] D \Psi+\frac{3 i K \cot \theta+i K^{3} \operatorname{Re} W e}{u_{s}-c} \Psi=0, \\
D^{2} \Psi+\frac{3 \Psi}{u_{s}-c}+K^{2} \Psi=0 .
\end{gathered}
$$

At $z=0$ the boundary conditions are

$$
\begin{gathered}
D^{3} \Psi-\left[i K \operatorname{Re}\left(u_{s}-c\right)+3 K^{2}\right] D \Psi+i K \operatorname{Re} D u_{s} \Psi=\left(\frac{i c K D a^{2} R e}{\phi}-1-2 K^{2} D a^{2}\right) D \Psi_{p}, \\
D \Psi-D a^{2} D \Psi_{p}=\frac{D a}{\alpha_{B J}}\left(D^{2} \Psi+K^{2} \Psi\right) \\
\Psi=D a^{2} \Psi_{p}
\end{gathered}
$$

while at $z=-d$ we have

$$
\Psi_{p}=0
$$

We now proceed by eliminating $\Psi_{p}(z)$ from this system as follows. Solving equation (3.36) we get

$$
\Psi_{p}(z)=c_{1} \cosh (K z)+c_{2} \sinh (K z)
$$

where $c_{1}$ and $c_{2}$ are arbitrary constants. From (3.41) and (3.42) we obtained

$$
c_{1}=-\frac{\Psi_{p}(0)}{D a^{2}} \text { and } c_{2}=\frac{\Psi_{p}(0)}{D a^{2}} \operatorname{coth}(K d),
$$

and we therefore have

$$
D \Psi_{p}(0)=\frac{K \operatorname{coth}(K d)}{D a^{2}} \Psi(0)
$$

This can be used to eliminate $\Psi_{p}(z)$ from (3.39) and (3.40). As a result, we then have the following problem for $\Psi(z)$

$$
\begin{gathered}
D^{4} \Psi-\left[i K \operatorname{Re}\left(u_{s}-c\right)+2 K^{2}\right] D^{2} \Psi+\left[i K^{3} \operatorname{Re}\left(u_{s}-c\right)+i K \operatorname{Re} D^{2} u_{s}+K^{4}\right] \Psi=0, \\
D^{3} \Psi-\left[i K \operatorname{Re}\left(u_{s}-c\right)+3 K^{2}\right] D \Psi+\frac{3 i K \cot \theta+i K^{3} \operatorname{Re} W e}{u_{s}-c} \Psi=0 \quad \text { at } z=h_{s} \\
D^{2} \Psi+\frac{3 \Psi}{u_{s}-c}+K^{2} \Psi=0 \quad \text { at } \quad z=h_{s}
\end{gathered}
$$




$$
\begin{gathered}
D^{3} \Psi-\left[i K \operatorname{Re}\left(u_{s}-c\right)+3 K^{2}\right] D \Psi+ \\
{\left[i K R e D u_{s}-\left(\frac{i c K D a^{2} R e}{\phi}-1-2 K^{2} D a^{2}\right) \frac{K}{D a^{2}} \operatorname{coth}(K d)\right] \Psi=0 \quad \text { at } z=0} \\
\frac{D a}{\alpha_{B J}} D^{2} \Psi-D \Psi+\left(\frac{D a}{\alpha_{B J}} K^{2}+K \operatorname{coth}(K d)\right) \Psi=0 \quad \text { at } z=0 .
\end{gathered}
$$

In order to carry out our stability analysis we must, for a given set of flow parameters $(R e, K a, D a, \phi$, $\left.\alpha_{B J}, d, \cot \theta\right)$, determine the growth rate of a particular perturbation distinguished by its wavenumber. More specifically, we must obtain the corresponding value of $c$. If $\Im(c)$ is negative the perturbation is damped, while if $\Im(c)$ is positive the perturbation is amplified. So, in dealing with the system given by equations (3.46) - (3.50) $c$ is also an unknown. We determine $c$ by requiring that nontrivial solutions exist for $\Psi$. Therefore, equations (3.46) - (3.50) comprise an eigenvalue problem with $c$ being the parameter to which characteristic values must be assigned.

\subsection{Numerical solution}

To calculate eigenvalues for the problem (3.46)-(3.50) we implement a Chebyshev collocation method. We first transform the domain $z \in\left(0, h_{s}\right)$ to $\xi \in(-1,1)$ by employing the transformation

$$
\xi=\frac{2}{h_{s}} z-1 .
$$

Consequently, the differential equation becomes

$$
\frac{16}{h_{s}^{4}} D_{\xi}^{4} \Phi-\frac{4}{h_{s}^{2}}\left[i K \operatorname{Re}\left(u_{s}-c\right)+2 K^{2}\right] D_{\xi}^{2} \Phi+\left[i K^{3} \operatorname{Re}\left(u_{s}-c\right)+i K \operatorname{Re} D_{\xi}^{2} u_{s}+K^{4}\right] \Phi=0,
$$

where $D_{\xi}$ is a differential operator denoting differentiation with respect to $\xi, \Phi(\xi)$ denotes $\Psi(z)$ in terms of $\xi$ and the equilibrium velocity in terms of $\xi$ is given by

$$
u_{s}=\left(-\frac{3}{8} \xi^{2}+\frac{3}{4} \xi+\frac{9}{8}\right) h_{s}^{2}+3 \frac{D a}{\alpha_{B J}} h_{s}+3 D a^{2} .
$$

The boundary conditions at $\xi=1$ are

$$
\begin{gathered}
\frac{8}{h_{s}^{3}} D_{\xi}^{3} \Phi-\frac{2}{h_{s}}\left[i K \operatorname{Re}\left(u_{s}-c\right)+3 K^{2}\right] D_{\xi} \Phi+\frac{3 i K \cot \theta+i K^{3} \operatorname{Re} W e}{u_{s}-c} \Phi=0, \\
\frac{4}{h_{s}^{2}} D_{\xi}^{2} \Phi+\frac{3 \Phi}{u_{s}-c}+K^{2} \Phi=0,
\end{gathered}
$$


and at $\xi=-1$ the boundary conditions are

$$
\begin{aligned}
& \frac{8}{h_{s}^{3}} D_{\xi}^{3} \Phi-\frac{2}{h_{s}}\left[i K R e\left(u_{s}-c\right)+3 K^{2}\right] D_{\xi} \Phi \\
&+\left[i K R e D_{\xi} u_{s}-\left(\frac{i c K D a^{2} R e}{\phi}-1-2 K^{2} D a^{2}\right) \frac{K}{D a^{2}} \operatorname{coth}(K d)\right] \Phi=0, \\
& \frac{4}{h_{s}^{2}} \frac{D a}{\alpha_{B J}} D_{\xi}^{2} \Phi-\frac{2}{h_{s}} D_{\xi} \Phi+\left(\frac{D a}{\alpha_{B J}} K^{2}+K \operatorname{coth}(K d)\right) \Phi=0 .
\end{aligned}
$$

We now expand the dependent variable in terms of Chebyshev polynomials as $\quad \Phi(\xi)=\sum_{j=1}^{N} a_{j} T_{j-1}(\xi)$ where $T_{n}(\xi)=\cos (n \arccos \xi), n=0,1,2, \ldots, N-1$. We discretise the $\xi$ interval and evaluate the differential equation at the interior grid points, and the boundary conditions at the end points. This yields an $N \times N$ algebraic system for the coefficients in the expansion, $\vec{a}=\left[a_{1}, a_{2}, \ldots a_{N}\right]^{T}$, which can be expressed in the form

$$
\vec{L} \vec{a}=c \vec{M} \vec{a}
$$

where $\vec{L}$ and $\vec{M}$ are $N \times N$ matrices which do not contain $c$. Characteristic values for the parameter $c$ were obtained numerically using the Matlab subroutine eig. However, this approach, i.e. solving the algebraic eigensystem, provides $N$ values for $c$, whereas the original system, (3.46) - (3.50) should have a single value. More specifically, a particular perturbation should have a single growth rate for a given set of flow parameters. To remedy this issue we proceed as follows. We calculate the eigenvalues of the algebraic system for different values of $N$ and find that for sufficiently large values, as $N$ is increased only one eigenvalue remains approximately constant. We select this value as the appropriate solution for $c$. In all the cases that we considered this technique was successful, with $N$ values between 10 and 15 proving to be sufficiently large.

To obtain results, for a given set of flow parameters, we iterate over the wavenumber and use our numerical method to calculate the temporal growth rate. A useful illustration of the results is given by the neutral stability curve in the $R e-k$ plane which indicates the wavenumber with zero growth rate as a function of the Reynolds number. This curve delineates the regions in the $R e-k$ plane corresponding to amplified or damped infinitesimal perturbations. The onset of instability for the flow corresponds to the critical Reynolds number, $R e_{\text {crit }}$, such that for smaller $R e$ values all wavenumbers have a non-positive growth rate, while for larger values there are wavenumbers with a positive growth rate indicating that the flow is unstable.

It is evident from the formulation of the problem (3.46) - (3.50) that the only effect of the length scale ratio, $\delta$, is to stretch the neutral stability curve in the $R e-k$ plane and thus does not affect the critical Reynolds number for the onset of instability. We thus present curves for $K=k \delta$ as a function of $R e$. We point out that $2 \pi / K$ is the wavelength of the perturbation scaled with the characteristic film thickness, $H$. In Figure 3.1 we present neutral stability curves for different Darcy numbers, including $D a=0$ which corresponds to the impermeable substrate case. It can be seen that for the smaller $D a$ values $R e_{\text {crit }}$ coincides with $R e_{0}$ which denotes the intercept with the Re-axis and corresponds to the 


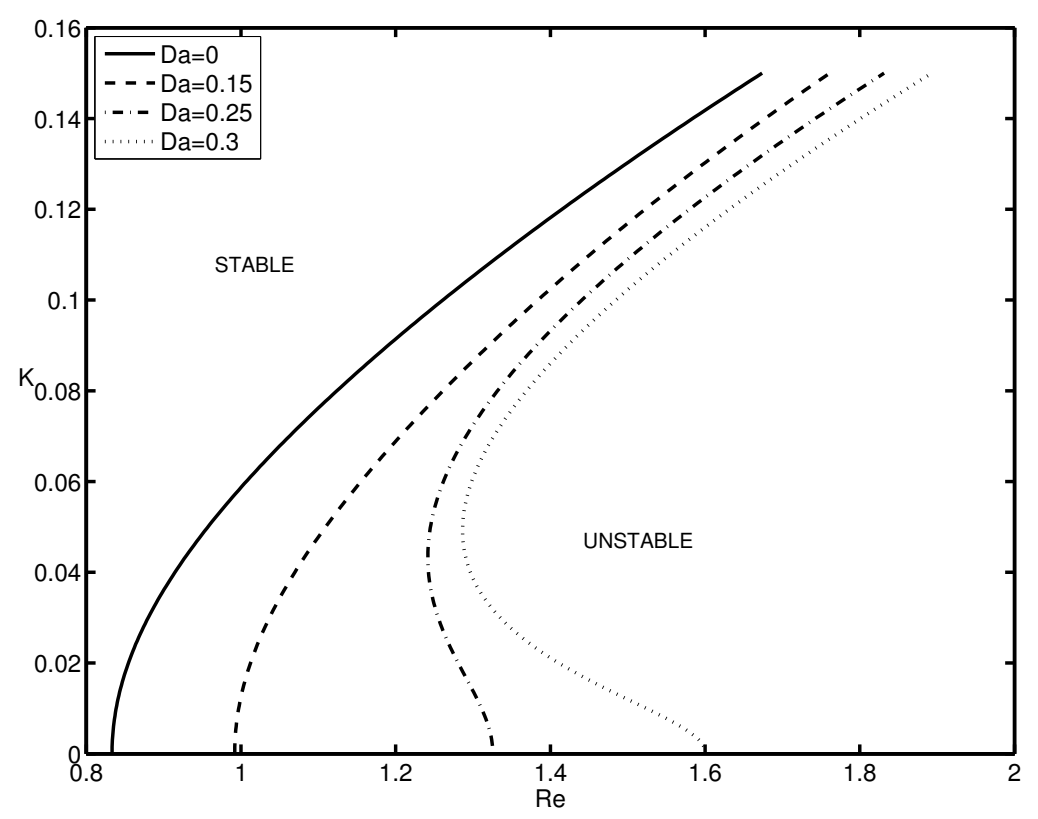

Figure 3.1: Neutral stability for different values of $D a$ with $\cot \theta=1, K a=100, \phi=0.01, \alpha_{B J}=2, d=$ 1.

onset of instability of infinitely long perturbations $(K=0)$. However, for the larger $D a$ values $R e_{\text {crit }}$ is smaller than $R e_{0}$. In other words, for sufficiently large permeability the onset of instability occurs by means of a perturbation of finite length. The possibility of unstable positive wavenumbers at the onset of surface instability has also been pointed out by Liu and Liu [16]. In the present work we investigate in detail how this phenomenon is related to various parameters, including surface tension, and to nonlinear effects.

As a verification of the numerical results we find that for the case $D a=0$ the obtained value for $R e_{\text {crit }}$ is a very close approximation of the accepted theoretical result $\frac{5}{6} \cot \theta[6,7]$. We have also obtained an asymptotic solution of the eigenvalue problem as $K \rightarrow 0$. More specifically, we consider perturbation expansions in the equations (3.46)-(3.50) of the form

$$
\begin{gathered}
\Psi(z)=\Psi_{0}(z)+K \Psi_{1}(z) \\
c=c_{0}+K c_{1},
\end{gathered}
$$

and obtain a hierarchy of problems for the different orders of $K$. For the $O(1)$ problem, we get

$$
D^{4} \Psi_{0}(z)=0
$$


with boundary conditions

$$
\begin{gathered}
D^{3} \Psi_{0}\left(h_{s}\right)=0, \quad\left(u_{s}\left(h_{s}\right)-c_{0}\right) D^{2} \Psi_{0}\left(h_{s}\right)+3 \Psi_{0}\left(h_{s}\right)=0 \\
D^{3} \Psi_{0}(0)=0, \quad \frac{D a}{\alpha_{B J}} D^{2} \Psi_{0}(0)-D \Psi_{0}(0)=0
\end{gathered}
$$

Solving this problem we get

$$
\Psi_{0}(z)=z^{2}+2 D a_{1} z \quad \text { and } \quad c_{0}=3 h_{s}^{2}+2 D a^{2}+6 D a_{1} h_{s},
$$

where $D a_{1}=\frac{D a}{\alpha_{B J}}$.

Now, collecting the terms in $K$ gives us

$$
D^{4} \Psi_{1}(z)-i \operatorname{Re}\left(u_{s}(z)-c_{0}\right) D^{2} \Psi_{0}(z)+i \operatorname{Re} D^{2} u_{s}(z) \Psi_{0}(z)=0
$$

with boundary conditions

$$
\begin{gathered}
D^{3} \Psi_{1}\left(h_{s}\right)-i \operatorname{Re}\left(u_{s}\left(h_{s}\right)-c_{0}\right) D \Psi_{0}\left(h_{s}\right)+\frac{3 i \cot \theta}{u_{s}\left(h_{s}\right)-c_{0}} \Psi_{0}\left(h_{s}\right)=0 \\
D^{2} \Psi_{1}\left(h_{s}\right)+\frac{3}{u_{s}\left(h_{s}\right)-c_{0}} \Psi_{1}\left(h_{s}\right)+\frac{3 c_{1}}{\left(u_{s}\left(h_{s}\right)-c_{0}\right)^{2}} \Psi_{0}\left(h_{s}\right)=0 \\
D^{3} \Psi_{1}(0)-i R e\left(u_{s}(0)-c_{0}\right) D \Psi_{0}(0)+\left[i R e D u_{s}(0)+\frac{1}{d D a^{2}}\right] \Psi_{0}(0)=0 \\
D a_{1} D^{2} \Psi_{1}(0)-D \Psi_{1}(0)+\frac{1}{d} \Psi_{0}(0)=0 .
\end{gathered}
$$

Solving this problem leads to the following expression for $c_{1}$

$$
\begin{aligned}
c_{1}=i\left[9 \operatorname{Reh}_{s}^{3} D a_{1}{ }^{3}+15 \operatorname{Reh}_{s}^{4} D a_{1}{ }^{2}-\frac{1}{5}\left(15 h_{s}^{2} \cot \theta-36 R e h_{s}^{5}\right) D a_{1}\right. \\
\left.-\frac{1}{5}\left(15 h_{s} \cot \theta+15 d \cot \theta\right) D a^{2}-\frac{1}{5}\left(5 h_{s}^{3} \cot \theta-6 R e h_{s}^{6}\right)\right] .
\end{aligned}
$$

Now, neutral stability is given by $\Im(c)=0$. Since $\Im\left(c_{0}\right)=0$, neutral stability is described by $\Im\left(c_{1}\right)=0$. Solving for $R e$ we obtain an expression that is independent of $K$. The prediction of this analysis, therefore, is that the neutral stability curve is a vertical line in the $R e-K$ plane. However, it must be pointed out that this result is valid as $K \rightarrow 0$. Therefore, we can only expect this threshold to be valid for small values of $K$. In other words, it is an approximation for the intercept of the neutral stability curve with the $R e$-axis. So, the asymptotic solution gives the following approximation for $R e_{0}$

$$
R e_{0}=\frac{5 \cot \theta\left(3 h_{s} D a^{2} \alpha_{B J}+3 h_{s}{ }^{2} D a+h_{s}{ }^{3} \alpha_{B J}+3 D a^{2} \alpha_{B J} d\right) \alpha_{B J}{ }^{2}}{3 h_{s}{ }^{3}\left(2 h_{s}{ }^{3} \alpha_{B J}{ }^{3}+12 h_{s}{ }^{2} D a \alpha_{B J}{ }^{2}+25 h_{s} D a^{2} \alpha_{B J}+15 D a^{3}\right)} .
$$




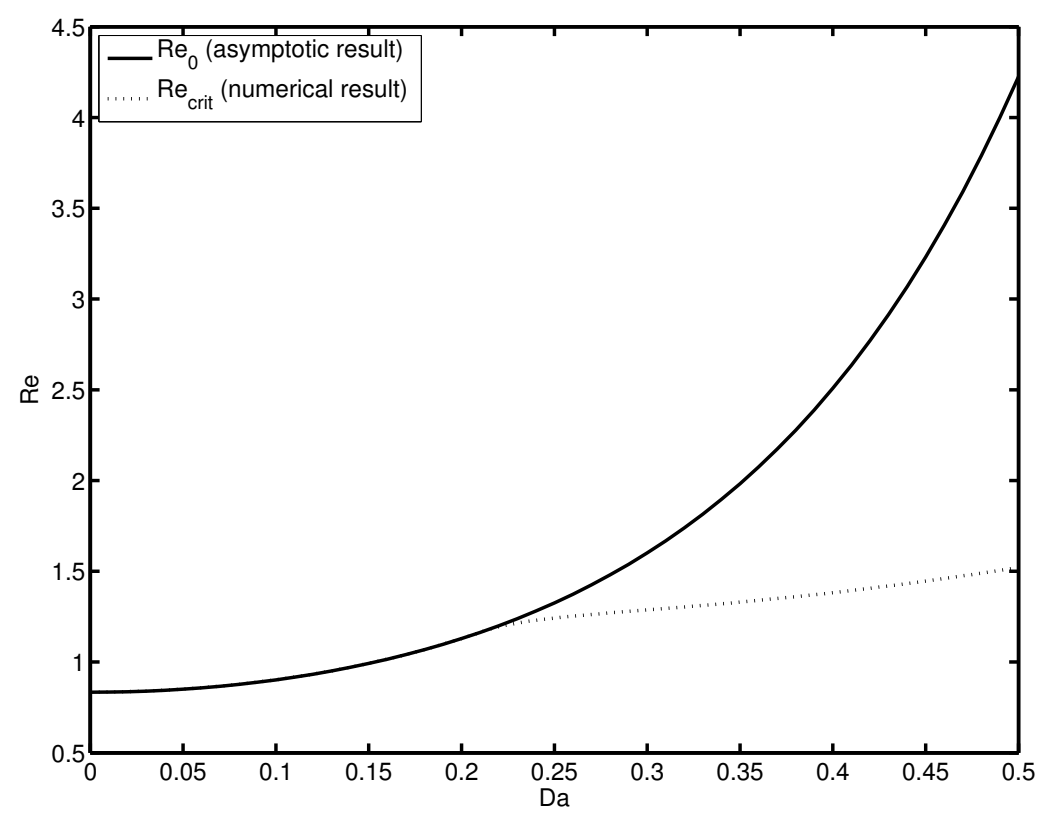

Figure 3.2: $R e_{\text {crit }}$ and $R e_{0}$ as functions of $D a$ with $\cot \theta=1, K a=100, \phi=0.01, \alpha_{B J}=2, d=1$.

This expression is in excellent agreement with the numerical results for $R e_{0}$. Figure 3.2 contains the numerically obtained critical Reynolds number as a function $D a$ together with the asymptotic expression for $R e_{0}$. The deviation of $R e_{\text {crit }}$ from $R e_{0}$ occurs for $D a$ values large enough for the onset of instability to be due to the amplification of a perturbation with positive wavenumber instead of the one with zero wavenumber. As it can be seen, in this range $R e_{0}$ is larger than $R e_{\text {crit }}$ with the difference increasing with $D a$. The indication thus is that the effect of bottom filtration is to stabilize the very long perturbations relative to the moderately long ones.

In Figure 3.3 we illustrate the effect of $d$, the scaled thickness of the porous substrate, on neutral stability. From these results we conclude that increasing the thickness of the substrate stabilizes the flow. Furthermore, it is evident that there is also a critical value of $d$ such that for smaller values infinitely long perturbations are unstable at the onset, while for larger values the onset of instability is related to a perturbation of finite wavelength.

Neutral stability curves for different Kapitza numbers are included in Figure 3.4. The curves have a common intercept with the $R e$-axis which is to be expected since the expression for $R e_{0}$ given in (3.58) is independent of $K a$. For the smaller Kapitza numbers $R e_{\text {crit }}$ is less than $R e_{0}$, however, it increases with $K a$ and beyond a certain value $R e_{c r i t}=R e_{0}$. For low to moderate surface tension $R e_{c r i t}<R e_{0}$ and the onset of instability is due to the amplification of a perturbation of finite wavelength. Surface tension affects waves of finite length, so it impacts the threshold for instability of the flow. In particular, increasing surface tension in this range of $K a$ values increases $R e_{c r i t}$ and thus acts to stabilize the 


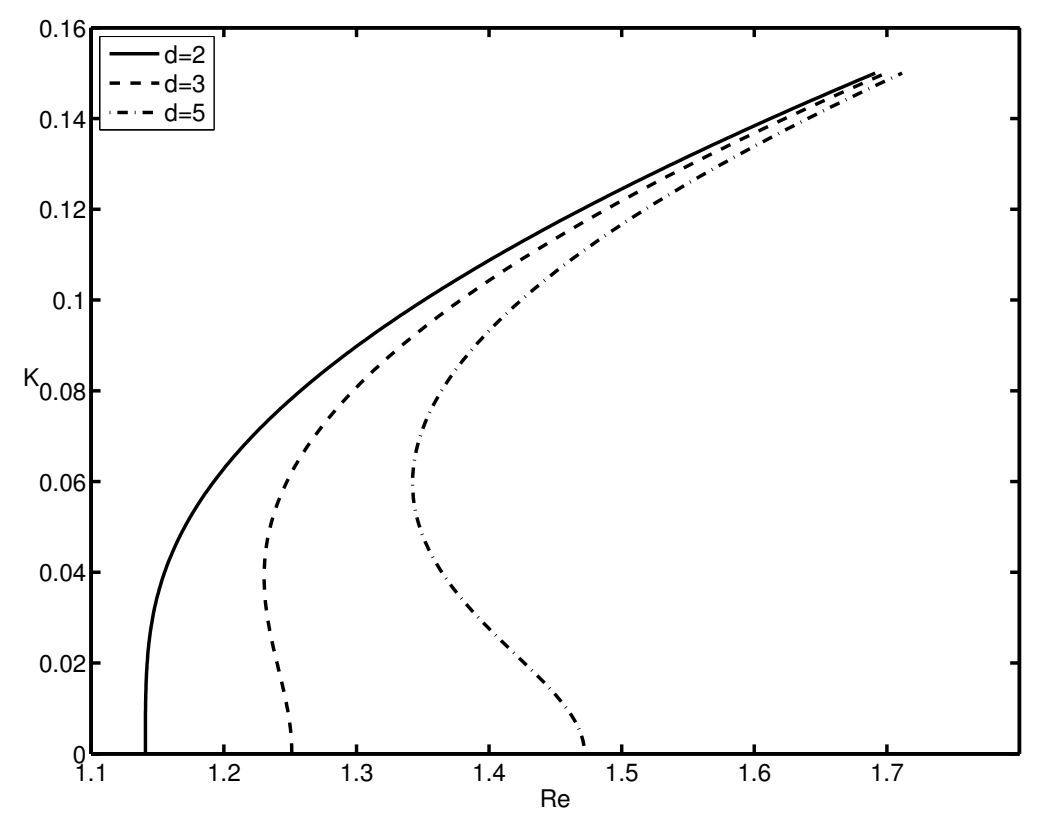

Figure 3.3: Neutral stability for different values of $d$ with $\cot \theta=1, K a=100, \phi=0.01, \alpha_{B J}=$ $0.1, D a=0.2$.

equilibrium flow. It is also apparent that the wavenumber of the most unstable mode at the onset is decreased with increasing surface tension. For strong surface tension $R e_{c r i t}=R e_{0}$, so infinitely long perturbations are more unstable and the onset of instability is independent of surface tension.

As it can be seen in Figure 3.5, varying $\phi$ has a similar effect to varying $K a$. Specifically, increasing $\phi$ stabilizes the flow when finite perturbations are amplified at the onset, and for sufficiently large $\phi$ values $R e_{\text {crit }}$ coincides with $R e_{0}$ in which case porosity does not affect the onset of instability. This is consistent with the asymptotic formula for $R e_{0}$ which is independent of $\phi$.

In Figures 3.6 - 3.8 we display the dependence of the critical Reynolds number on the permeability of the substrate for different values of the other flow parameters. Liu and Liu [16], in considering very low permeability, have found it to destabilize the surface of the film for small values of $\alpha_{B J}$. We considered a wider range of Darcy numbers and have found that, depending on the value of other parameters, an increase in bottom permeability can also stabilize the flow. Figure 3.6 shows the graphs of $R e_{\text {crit }}$ as a function of $D a$ for different values of $\alpha_{B J}$. It can be seen that for a given $D a$ value $R e_{\text {crit }}$ increases with $\alpha_{B J}$. This fact is also evident in the results obtained by Liu and Liu [16]. An increase in $\alpha_{B J}$ means less slip at the bottom of the fluid film resulting in a slower flow rate thus stabilizing the flow. It is also interesting to note that there is a critical value of $\alpha_{B J}$ such that for larger $\alpha_{B J}$ values $R e_{\text {crit }}$ changes from a decreasing function to an increasing function of $D a$. Figures 3.7 and 3.8 illustrate the effect of $\phi$ and $d$ on the variation of $R e_{c r i t}$ with $D a$. It is apparent that sufficiently large values of these parameters result in non-monotonic variations of $R e_{c r i t}$ with $D a$. 


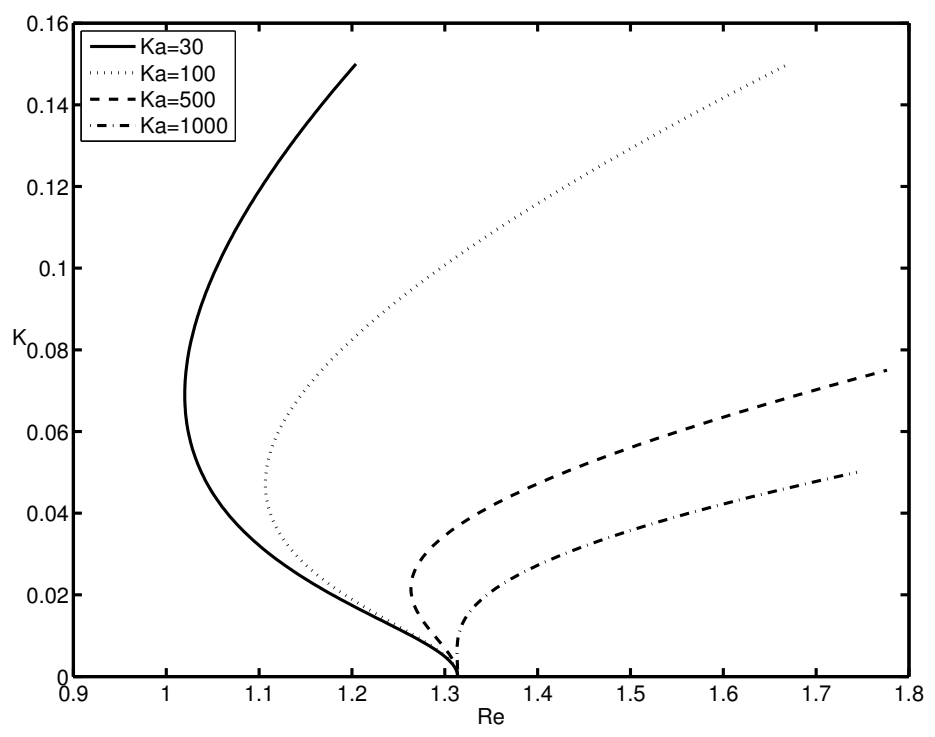

Figure 3.4: Neutral stability for different values of $K a$ with $\cot \theta=1, d=1, \phi=0.01, \alpha_{B J}=1, D a=$ 0.3 .

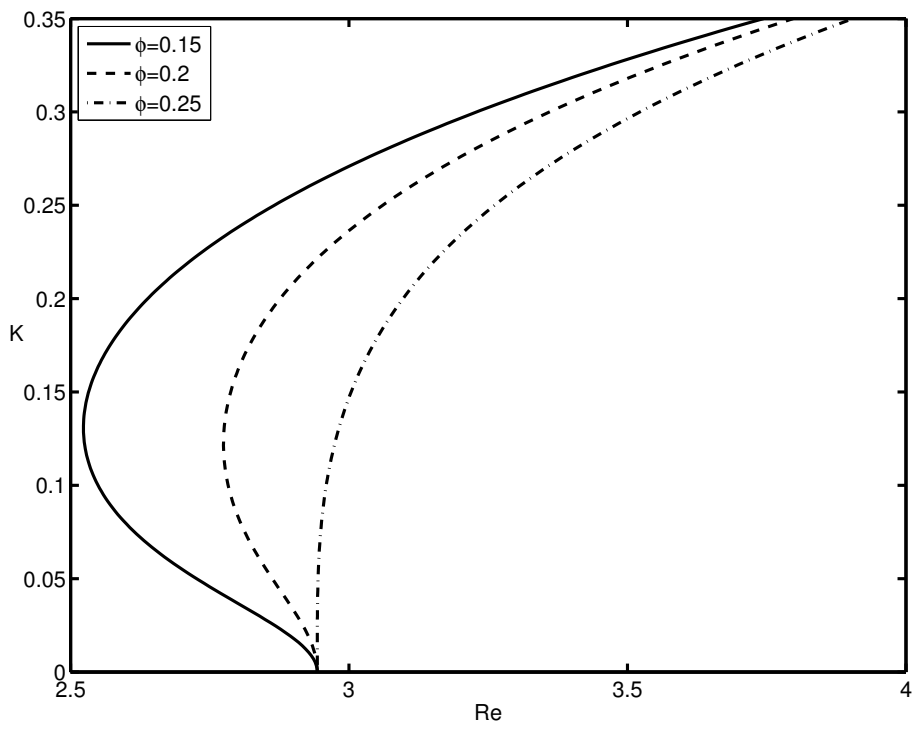

Figure 3.5: Neutral stability for different values of $\phi$ with $\cot \theta=1, d=3, K a=100, \alpha_{B J}=1, D a=0.4$. 


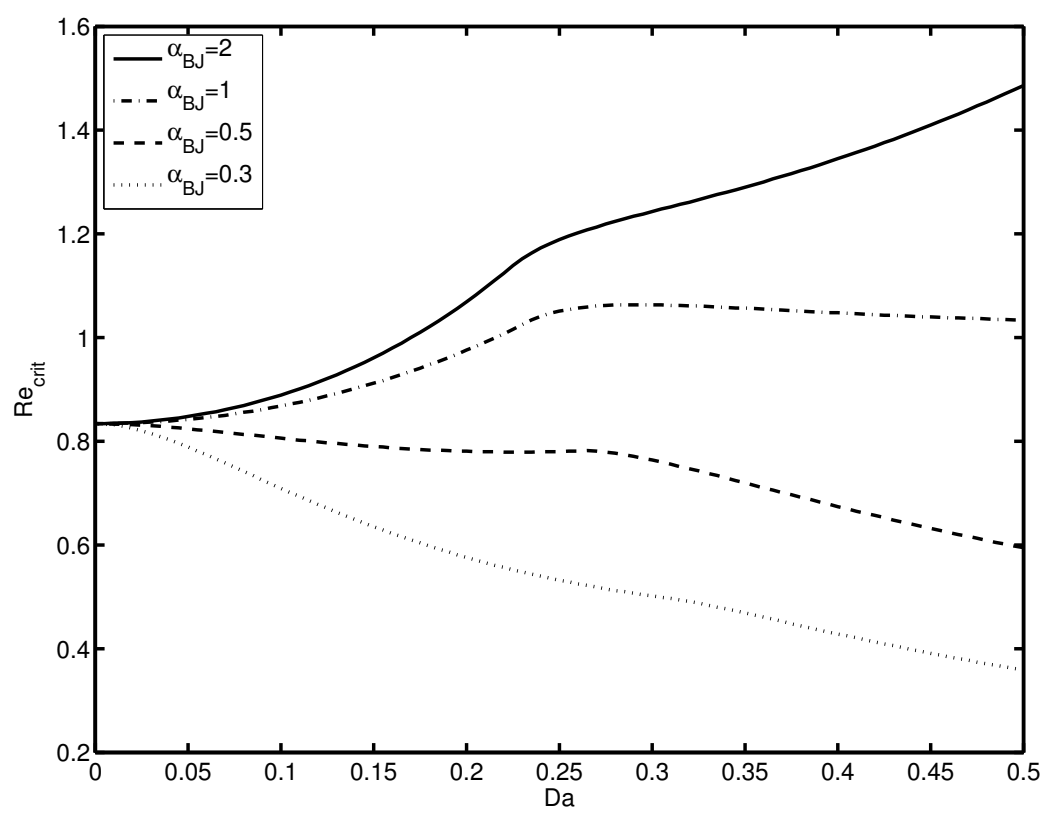

Figure 3.6: The critical Reynolds number as a function of $D a$ for different values of $\alpha_{B J}$ with $\cot \theta=$ $1, d=0.5, \phi=0.01, K a=100$.

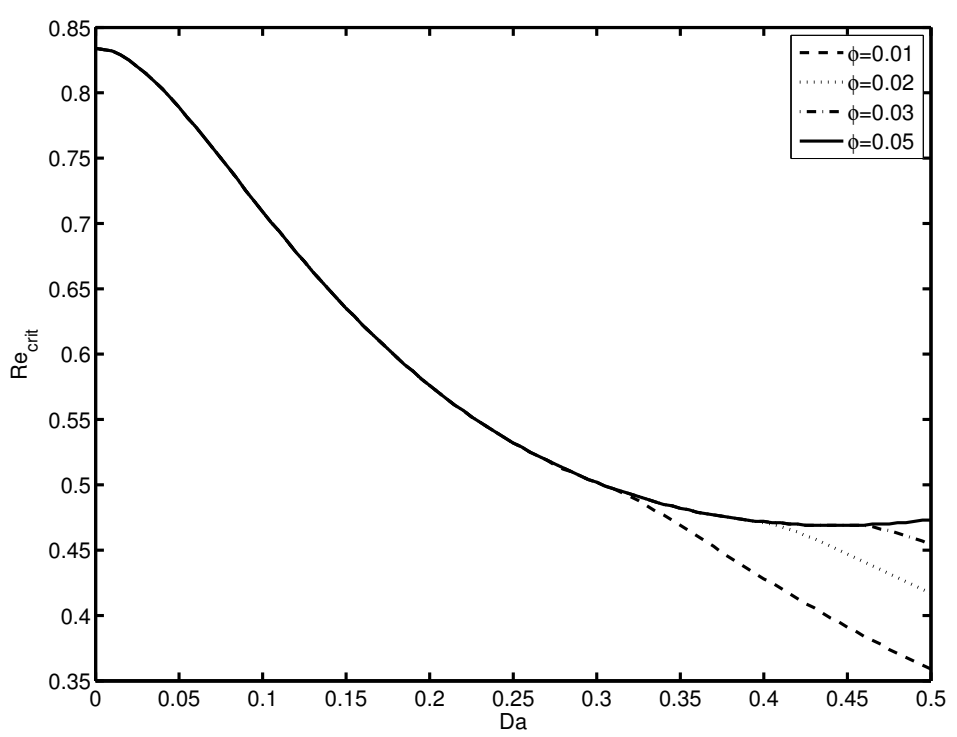

Figure 3.7: The critical Reynolds number as a function of $D a$ for different values of $\phi$ with $\cot \theta=1, d=$ $0.5, \alpha_{B J}=0.3, K a=100$. 


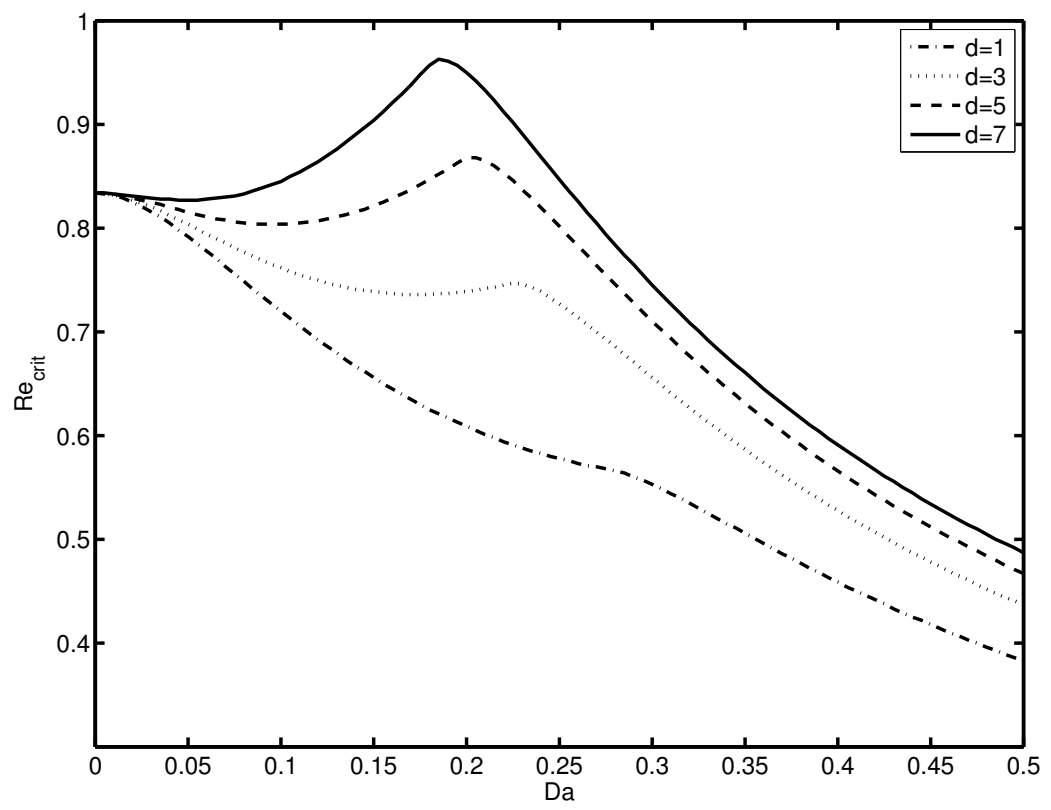

Figure 3.8: The critical Reynolds number as a function of $D a$ for different values of $d$ with $\cot \theta=1, \phi=$ $0.01, \alpha_{B J}=0.3, K a=100$.

In Figures 3.9 and 3.10 we show the temporal growth rate and phase speed of perturbations as functions of the scaled wavenumber, $K$, for supercritical Reynolds numbers close to the critical value for the onset of instability. The results in Figure 3.9 correspond to the impermeable bottom case, while in Figure 3.10 we have a case with bottom filtration. The growth rate distributions with $K$ for the case with filtration reveal that just beyond criticality a band of moderately long perturbations are being amplified while all other wavelengths, including the very long ones, are being damped. As the Reynolds number is increased, eventually the very long perturbations also become unstable and the growth rate curve resembles that for the impermeable substrate case. In both cases the wavenumber with the fastest growth rate increases with $R e$.

In the impermeable bottom case the phase speed decreases with $K$, while if filtration is present the phase speed is a non-monotonic function attaining a maximum value on the range of unstable wavenumbers. It appears that the wavenumber corresponding to the maximum phase speed does not coincide with the one where the maximum growth rate is attained. In fact, the maximum phase speed decreases with $R e$, as does the $K$ value at which it is attained. 

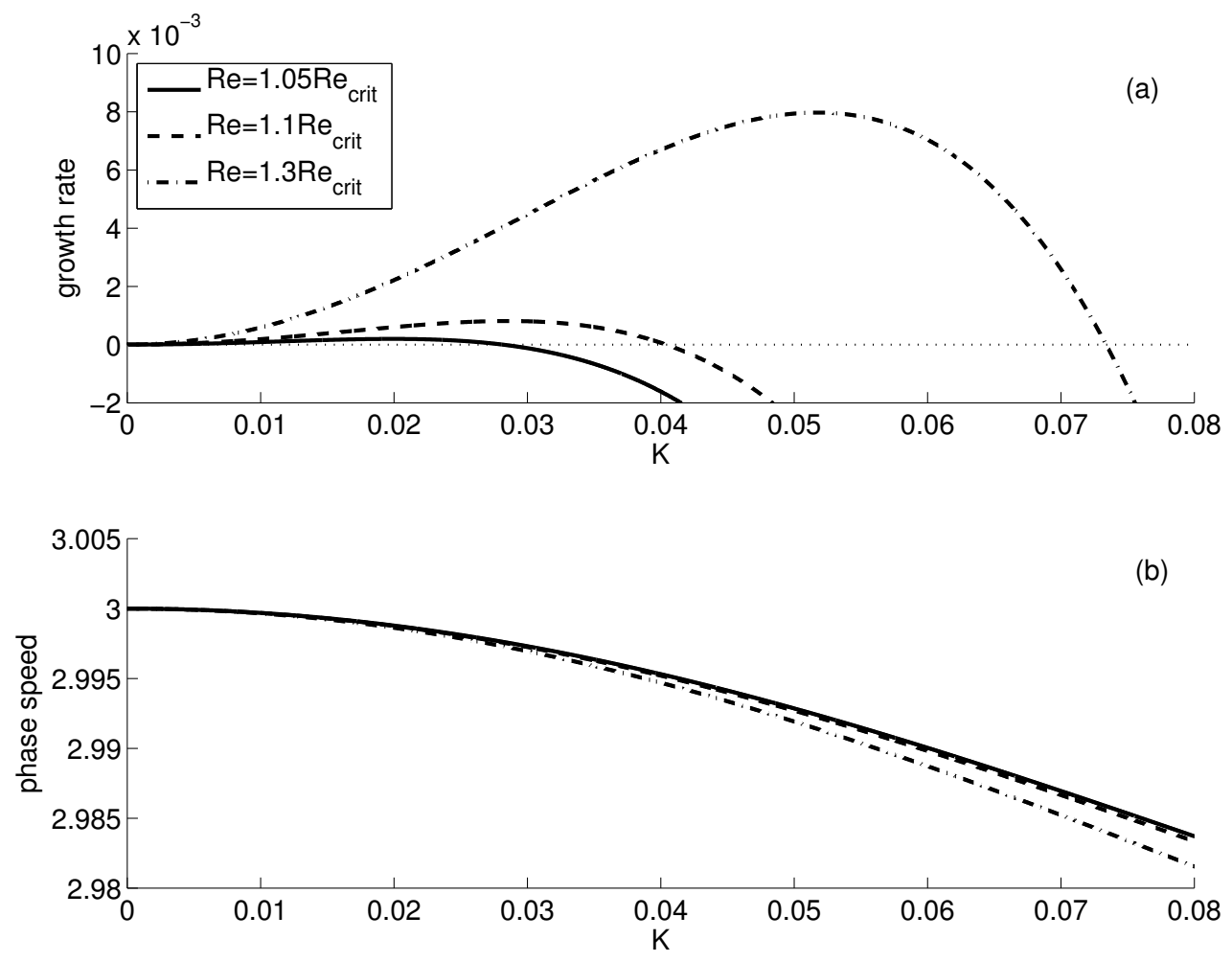

Figure 3.9: The temporal growth rate and phase speed as functions of $K$ for the impermeable bottom case $(D a=0)$, with $\cot \theta=1, K a=100$. In this case $R e_{\text {crit }}=5 / 6$. 

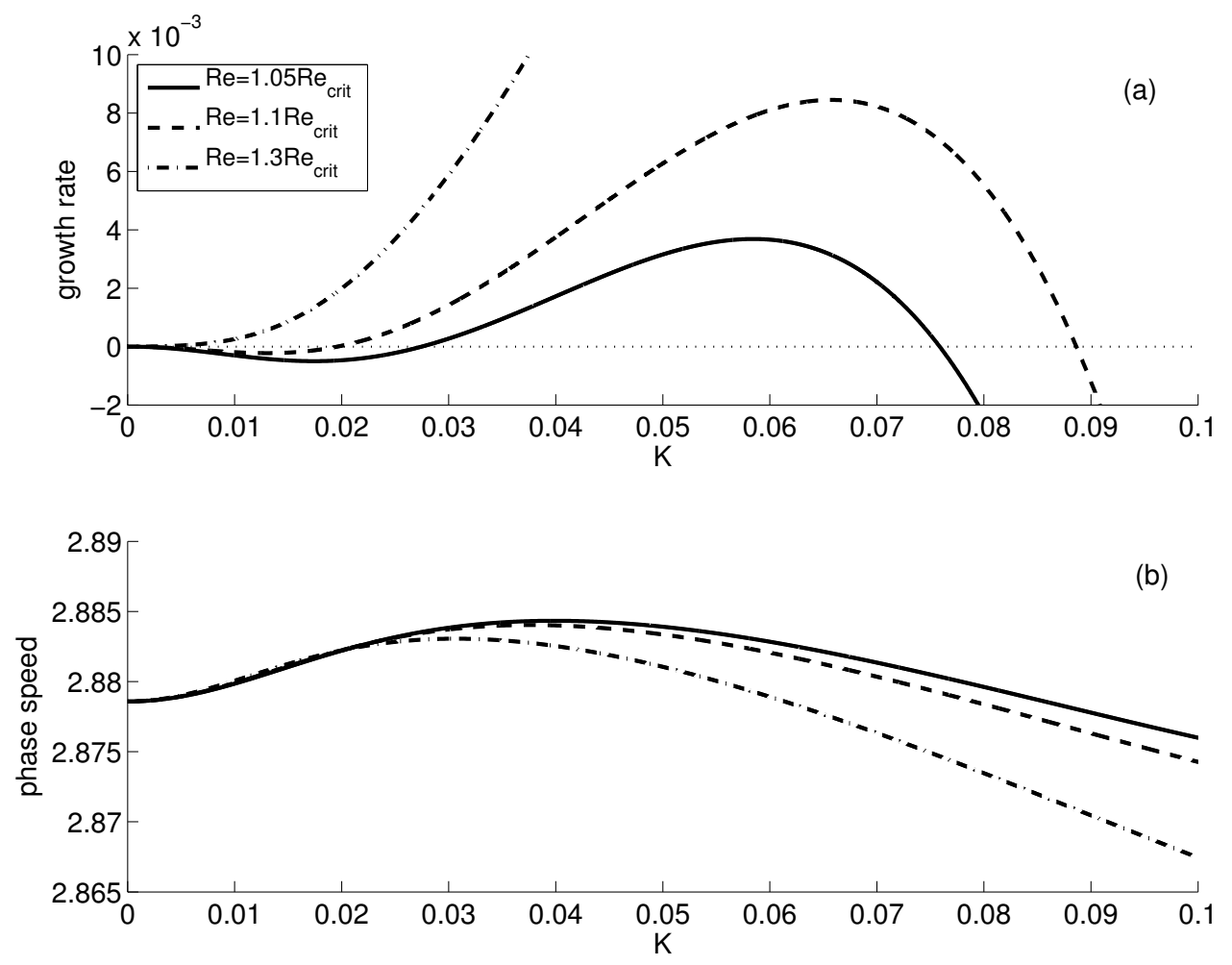

Figure 3.10: The temporal growth rate and phase speed as functions of $K$ for $D a=0.3, \cot \theta=1, \phi=$ $0.01, d=1, \alpha_{B J}=2, K a=100$. In this case $R e_{\text {crit }} \approx 1.286$. 


\section{Chapter 4}

\section{Nonlinear Effects}

Our goal in this chapter is to include nonlinear effects into solutions obtained for our governing equations. To accomplish this we employ certain appropriate approximations that simplify the equations. We first obtain what is referred to as the long-wave equations by discarding terms based on the assumed smallness of the length to thickness aspect ratio, $\delta$. These equations can be further simplified by applying a weighted-residual method. Numerical solutions can be effectively obtained for the resulting "reduced model".

\subsection{Long-wave equations}

As it is detailed in chapter 2, the results from the full equations indicated that instability is related to infinitely long perturbations or perturbations that have a finite wavelength but are still long relative to the thickness of the film. We thus expect to obtain an accurate approximation of the stability analysis by implementing a simplified model based on the smallness of the aspect ratio $\delta$. We proceed by considering the asymptotic limit $\delta \rightarrow 0$ and deem $O\left(\delta^{2}\right)$ terms as negligible. We assume all the parameters of the problem to be $O(1)$ with the exception of $K a$ which is assumed to be large enough so that $K a \delta^{3}$ is not

negligible, and discard the $O\left(\delta^{2}\right)$ terms in the full equations (2.14) - (2.26). This allows us to eliminate the pressure from the system as follows. The $z$-momentum equation, (2.16) becomes

$$
\frac{\partial p}{\partial z}=\frac{1}{R e}\left(\delta \frac{\partial^{2} w}{\partial z^{2}}-3 \cot \theta\right)
$$

Integrating this equation with respect to $z$ results in

$$
p=\left.p\right|_{z=h_{s}}-\frac{1}{R e}\left(\left.\delta \frac{\partial w}{\partial z}\right|_{z=h_{s}}-\delta \frac{\partial w}{\partial z}-3 \cot \theta\left(h_{s}-z\right)\right) .
$$


We get $\left.p\right|_{z=h_{s}}$ from the normal force condition (2.20)

$$
\left.p\right|_{z=h_{s}}=\frac{2}{R e}\left(\left.\delta \frac{\partial w}{\partial z}\right|_{z=h_{s}}-\left.\delta \frac{\partial h}{\partial x} \frac{\partial u}{\partial z}\right|_{z=h_{s}}\right)-\delta^{2} W e \frac{\partial^{2} h}{\partial x^{2}} .
$$

The expression for $p$ is introduced into the $x$-momentum equation, (2.15). Similarly, we can eliminate the filtration pressure. Specifically, we integrate (2.19) to obtain

$$
p_{p}=\left.p_{p}\right|_{z=0}-3 \delta \cot \theta z
$$

The expression for $\left.p_{p}\right|_{z=0}$ is obtained from condition (2.23) which, in our asymptotic limit, is given by $p_{p}=\delta \operatorname{Rep}$ at $z=0$. The expression for $p_{p}$ is then introduced into equation (2.18).

The complete set of long-wave equations is given by

$$
\begin{gathered}
\frac{\partial u}{\partial x}+\frac{\partial w}{\partial z}=0 \\
\delta R e\left(\frac{\partial u}{\partial t}+u \frac{\partial u}{\partial x}+w \frac{\partial u}{\partial z}\right)=-3 \delta \cot \theta \frac{\partial h}{\partial x}+\delta^{3} \operatorname{ReWe} \frac{\partial^{3} h}{\partial x^{3}}+3+\frac{\partial^{2} u}{\partial z^{2}}, \\
\frac{\partial u_{p}}{\partial x}+\frac{\partial w_{p}}{\partial z}=0 \\
\frac{D a^{2} \operatorname{Re} \delta}{\phi} \frac{\partial u_{p}}{\partial t}=-3 \delta \cot \theta \frac{\partial h}{\partial x}+\delta^{3} \operatorname{ReWe} \frac{\partial^{3} h}{\partial x^{3}}-u_{p}+3
\end{gathered}
$$

subjected to the conditions

$$
\begin{gathered}
\frac{\partial u}{\partial z}=0 \quad \text { at } z=h(x, t), \\
w=\frac{\partial h}{\partial t}+u \frac{\partial h}{\partial x} \quad \text { at } z=h(x, t), \\
w=D a^{2} w_{p} \quad \text { at } z=0, \\
\frac{\partial u}{\partial z}=\frac{\alpha_{B J}}{D a}\left(u-D a^{2} u_{p}\right) \text { at } z=0, \\
w_{p}=0 \text { at } z=-d .
\end{gathered}
$$

For the purpose of gauging the accuracy of this long-wave model, we perform a linear stability analysis and compare the results with those from the full equations. We perturb the primitive variables and linearize the governing equations. We then convert to the stream function formulation and introduce normal modes thus obtaining

$$
D^{3} \Psi-\left[i K \operatorname{Re}\left(u_{s}-c\right)\right] D \Psi+i K \operatorname{Re} D u_{s} \Psi-K_{1} \eta=0,
$$

where $\Psi(z)$ is the amplitude of the stream function perturbation, $\eta$ is the amplitude of the perturbation added to the thickness of the fluid film and $K_{1}=3 i K \cot \theta+i K^{3} R e W e$. The associated boundary 
conditions are

$$
\begin{gathered}
D^{2} \Psi-3 \eta=0 \quad \text { at } z=h_{s}, \\
\Psi-\left(c-u_{s}\right) \eta=0 \text { at } z=h_{s}, \\
\left(1-K_{2} c\right) \Psi+d D a^{2} K_{1} \eta=0 \quad \text { at } z=0, \\
\left(1-K_{2} c\right) D \Psi+D a^{2} K_{1} \eta-\frac{D a}{\alpha_{B J}}\left(1-K_{2} c\right) D^{2} \Psi=0 \text { at } z=0,
\end{gathered}
$$

where $K_{2}=i K D a^{2} R e / \phi$.

As it is exemplified by the results in Figures 4.1 and 4.2, the predictions made by the long-wave equations are in good agreement with those from the full equations. Most notably, the long-wave equations capture the instability of moderately long perturbations associated with the onset of instability of the flow.

\subsection{The weighted-residual method}

To study nonlinear effects on the stability of the flow we effectuate a further simplification of the longwave equations by eliminating the explicit dependence on the vertical coordinate. Now, the equation for the horizontal filtration velocity, equation (4.7), is free of $z$ derivatives, so we assume that $u_{p}$ is $z$ independent, i.e. $u_{p}=u_{p}(x, t)$. Then, upon integrating the continuity equation in the porous medium, (4.6), we obtain the vertical filtration velocity

$$
w_{p}=-d \frac{\partial u_{p}}{\partial x} .
$$

Integrating the continuity equation for the fluid film, (4.4), with respect to $z$ yields

$$
\frac{\partial h}{\partial t}+\frac{\partial}{\partial x}\left(q+d D a^{2} u_{p}\right)=0
$$

where

$$
q(x, t)=\int_{0}^{h(x, t)} u(x, z, t) d z .
$$

In order to eliminate the $z$ dependence from equation (4.5) we resort to a weighted-residual method. We introduce the function

$$
b(x, z, t)=2 h z-z^{2}+2 D a_{1} h+\frac{2}{3} D a^{2} u_{p},
$$

where $D a_{1}=\frac{D a}{\alpha_{B J}}$. This function satisfies the boundary condition for $u$ (4.8), as well as the linear approximation in $D a$ of the boundary condition (4.11). We thus employ it as a base function and consider $u$ expressed as

$$
u=\frac{3 q}{2\left(h^{3}+3 D a_{1} h^{2}+D a^{2} h u_{p}\right)} b .
$$

The coefficient of $b$ in this expansion is such that $q$ is introduced as a new dependent variable and is 


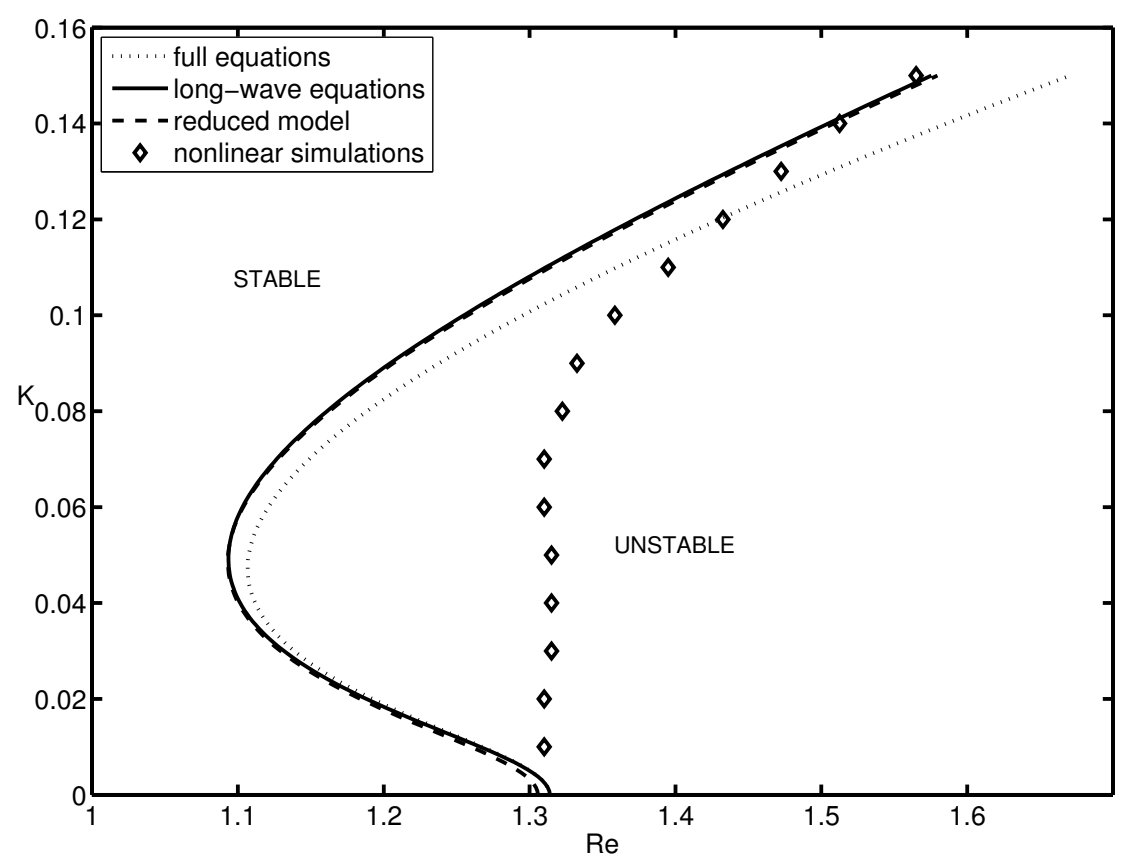

Figure 4.1: Neutral stability obtained by the different methods with $D a=0.3, \cot \theta=1, K a=100, \phi=$ $0.01, \alpha_{B J}=1, d=1$.

connected to $u$ through the relation (4.19).

In accordance with the Galerkin approach we introduce expression (4.20) into equation (4.5), multiply by $b$ and integrate with respect to $z$ from 0 to $h$. It should be pointed out that the assumed expression for $u$ does not in fact satisfy the boundary condition (4.11). We rectify this by applying integration by parts to the last term in (4.5) and substituting the correct boundary condition into the boundary terms. More specifically, we use

$$
\int_{0}^{h} b \frac{\partial^{2} u}{\partial z^{2}} d z=\left[\frac{\partial b}{\partial z}\left(D a_{1} \frac{\partial u}{\partial z}+D a^{2} u_{p}\right)-b \frac{\partial u}{\partial z}\right]_{z=0}+\int_{0}^{h} \frac{\partial^{2} b}{\partial z^{2}} u d z
$$

into which we then substitute expression (4.20) for $u$. Setting the residual of equation (4.5) to zero gives

$$
\begin{aligned}
\frac{\partial q}{\partial t}+\frac{\partial f}{\partial x} & =s_{1}+s_{2} \delta^{2} W e \frac{\partial^{3} h}{\partial x^{3}}+ \\
& \frac{q^{2}}{14 \Theta_{1}^{2} \Theta_{2}^{2}}\left(s_{3} \frac{\partial h}{\partial x}+s_{4} \frac{\partial u_{p}}{\partial x}\right)+\frac{q}{R e \Theta_{1} \Theta_{2}}\left(s_{5} \frac{\partial h}{\partial x}+s_{6} \frac{\partial u_{p}}{\partial x}\right)+\frac{\Theta_{1}}{\operatorname{Re} \Theta_{2}}\left(s_{7} \frac{\partial h}{\partial x}+s_{8} \frac{\partial u_{p}}{\partial x}\right),
\end{aligned}
$$

where

$$
\Theta_{1}=D a^{2} u_{p}+h^{2}+3 h D a_{1}
$$




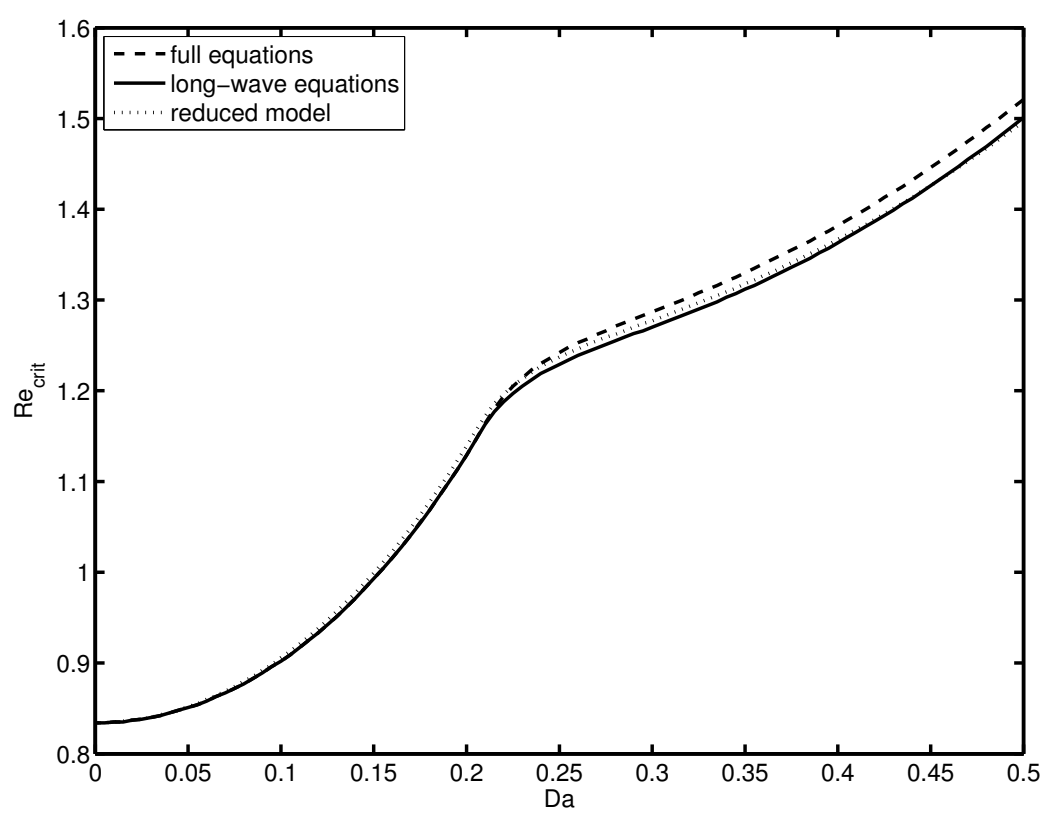

Figure 4.2: The critical Reynolds number obtained by the different methods with $\cot \theta=1, \phi=$ $0.01, \alpha_{B J}=2, K a=100$ and $d=1$.

$$
\begin{gathered}
\Theta_{2}=5 D a^{4} u_{p}{ }^{2}+30 D a_{1} u_{p} h D a^{2}+10 u_{p} h^{2} D a^{2}+30 h^{3} D a_{1}+45 h^{2} D a_{1}{ }^{2}+6 h^{4} . \\
f=\frac{q^{2}}{14 h \Theta_{1} \Theta_{2}}\left(1890 h^{3} D a_{1}{ }^{3}+\right. \\
1890 h^{4} D a_{1}{ }^{2}+1890 h^{2} u_{p} D a_{1}{ }^{2} D a^{2}+714 h^{5} D a_{1}+1260 u_{p} h^{3} D a_{1} D a^{2} \\
\left.+630 h u_{p}{ }^{2} D a_{1} D a^{4}+102 h^{6}+231 D a^{2} u_{p} h^{4}+210 D a^{4} u_{p}{ }^{2} h^{2}+70 D a^{6} u_{p}{ }^{3}\right) \\
s_{1}=\frac{1}{R e \Theta_{1} \Theta_{2} \delta}\left(30 D a^{6} u_{p}{ }^{3} h+225 D a^{4} u_{p}{ }^{2} h^{2} D a_{1}+75 D a^{4} u_{p}{ }^{2} h^{3}-30 D a^{4} q u_{p}{ }^{2}-\phi q h^{4} u_{p}\right. \\
+540 D a^{2} u_{p} h^{3} D a_{1}{ }^{2}+360 D a^{2} u_{p} h^{4} D a_{1}-45 D a^{2} q u_{p} h^{2}-135 D a^{2} u_{p} q h D a_{1}+ \\
60 D a^{2} u_{p} h^{5}+3 \phi q h^{4}-90 q h^{3} D a_{1}-135 q h^{2} D a_{1}{ }^{2}+ \\
\left.135 h^{6} D a_{1}+405 h^{5} D a_{1}{ }^{2}+405 h^{4} D a_{1}{ }^{3}+15 h^{7}-15 q h^{4}\right) \\
s_{2}=\frac{h}{\Theta_{1} \Theta_{2}}\left(5 D a^{6} u_{p}{ }^{3}+45 h u_{p}{ }^{2} D a_{1} D a^{4}+15 D a^{4} u_{p}{ }^{2} h^{2}+135 h^{2} u_{p} D a_{1}{ }^{2} D a^{2}+\right. \\
\left.90 u_{p} h^{3} D a_{1} D a^{2}+15 D a^{2} u_{p} h^{4}+\phi q h^{3}+5 h^{6}+135 h^{4} D a_{1}{ }^{2}+135 h^{3} D a_{1}{ }^{3}+45 h^{5} D a_{1}\right)
\end{gathered}
$$




$$
\begin{gathered}
s_{3}=-h^{2}\left(1260 D a^{6} u_{p}{ }^{3} h D a_{1}+2835 D a^{4} u_{p}{ }^{2} h^{2} D a_{1}{ }^{2}-360 D a^{4} u_{p}{ }^{2} h^{3} D a_{1}-3105 D a^{2} u_{p} h^{4} D a_{1}{ }^{2}+\right. \\
1890 D a^{2} u_{p} h^{3} D a_{1}{ }^{3}-1908 D a^{2} u_{p} h^{5} D a_{1}+175 D a^{8} u_{p}{ }^{4}+105 D a^{6} u_{p}{ }^{3} h^{2}-282 D a^{4} u_{p}{ }^{2} h^{4}- \\
\left.282 D a^{2} u_{p} h^{6}-36 h^{8}-504 h^{7} D a_{1}-2178 h^{6} D a_{1}{ }^{2}-3240 h^{5} D a_{1}{ }^{3}\right), \\
s_{4}=-h^{2}\left(-135 h^{5} D a_{1}{ }^{2} D a^{2}-945 u_{p}{ }^{2} h^{2} D a_{1} D a^{6}-720 u_{p} h^{4} D a_{1} D a^{4}-\right. \\
1890 h^{3} u_{p} D a_{1}{ }^{2} D a^{4}-72 D a^{4} u_{p} h^{5}-140 h D a^{8} u_{p}{ }^{3}- \\
\left.225 D a^{6} u_{p}{ }^{2} h^{3}-945 h^{4} D a_{1}{ }^{3} D a^{2}+120 D a^{2} h^{6} D a_{1}+30 D a^{2} h^{7}\right), \\
s_{5}=-3 \phi h^{4} \cot \theta, \\
s_{6}=\frac{D a^{2}}{8}\left(20 D a^{4} u_{p}{ }^{2} h R e d-120 D a^{4} u_{p}{ }^{2} R e d D a_{1}+57 D a^{2} u_{p} h^{3} R e d-\right. \\
\left.720 D a^{2} u_{p} h \operatorname{Red} D a_{1}{ }^{2}+147 h^{4} R e d D a_{1}+21 h^{5} R e d-180 h^{3} R e d D a_{1}{ }^{2}-1080 h^{2} R e d D a_{1}{ }^{3}\right), \\
s_{7}=-5\left(3 h \cot \theta u_{p} D a^{2}+3 h^{3} \cot \theta+9 h^{2} \cot \theta D a_{1}\right) \\
s_{8}=-5\left(\operatorname{Red} u_{p}{ }^{2} D a^{6}+3 h \operatorname{Red} D a_{1} u_{p} D a^{4}\right) .
\end{gathered}
$$

Equations (4.18), (4.21) and (4.7) constitute our reduced model and govern the dependent variables $h(x, t), q(x, t)$ and $u_{p}(x, t)$, which are the thickness of the fluid film, the flow rate of the film and the filtration velocity in the substrate, respectively. If we set $D a=0$ then equations (4.18) and (4.21) reduce to the modified integrated-boundary-layer equations proposed by Ruyer-Quil and Manneville [23] for flow over an impermeable surface. These equations predict the correct threshold for the onset of instability and it has been demonstrated [24] that they constitute the optimal weighted-residual approximation. For the permeable substrate case such an analysis of equations (4.18), (4.21) and (4.7) would be quite complicated. Therefore, to gauge the accuracy of our reduced model we determine the effect of the weighted-residual approximation on the linear stability results.

Adding normal-mode perturbations to the equilibrium solution gives

$$
h=h_{s}+\tilde{h} e^{\sigma t} e^{i k x}, q=1+\tilde{q} e^{\sigma t} e^{i k x}, u_{p}=3+\tilde{u_{p}} e^{\sigma t} e^{i k x} .
$$

Substituting the perturbed equilibrium solution into our reduced model and linearizing with respect to the amplitudes $\tilde{h}, \tilde{q}$ and $\tilde{u_{p}}$ leads to a dispersion relation. This equation was obtained with the aid of the Maple Computer Algebra System, however its formulation is quite lengthy and it is pointless to present it here. Suffice it to say that it is cubic in $\sigma$ and for fixed values of the other parameters the solutions were obtained by means of the Matlab polynomial root finder. The real part of $\sigma$ measures the temporal growth rate of the perturbation, so we are able to determine neutral stability. As it is illustrate in Figures 4.1 and 4.2, the results are in excellent agreement with those from the long-wave equations for a wide range of permeability values. There is thus strong evidence that the reduced model 
is accurate, and we carry out a nonlinear stability analysis on it.

The reduced model is amenable to a very effective numerical method. The equations constitute a system of nonlinear hyperbolic conservation laws with source terms. Terms which cannot be expressed as total derivatives, and thus cannot be included in the flux, appear as source terms involving the derivatives of the state variables. We deal with these terms by following a fractional-step approach. Such a method was applied in previous investigations of inclined film flow $[13,25,26]$. With this procedure, in order to advance the solution from one time level to the next, we apply a two-step process. In the first step we discard the derivative source terms and consider the system

$$
\begin{gathered}
\frac{\partial h}{\partial t}+\frac{\partial}{\partial x}\left(q+d D a^{2} u_{p}\right)=0, \\
\frac{\partial q}{\partial t}+\frac{\partial f}{\partial x}=s_{1}, \\
\frac{D a^{2} R e \delta}{\phi} \frac{\partial u_{p}}{\partial t}+3 \delta \cot \theta \frac{\partial h}{\partial x}=3-u_{p},
\end{gathered}
$$

which is of the general form

$$
\frac{\partial \mathbf{V}}{\partial t}+\frac{\partial}{\partial x} \mathbf{F}(\mathbf{V})=\mathbf{B}(\mathbf{V})
$$

where $\mathbf{V}, \mathbf{F}$ and $\mathbf{B}$ are column vectors with $\mathbf{V}$ containing the dependent variables of the system. We solved this system using the extension to MacCormack's scheme made by LeVeque and Yee [27] to include source terms via the scheme

$$
\begin{gathered}
\mathbf{V}_{j}^{*}=\mathbf{V}_{j}^{n}-\frac{\Delta t}{\Delta x}\left[\mathbf{F}\left(\mathbf{V}_{j+1}^{n}\right)-\mathbf{F}\left(\mathbf{V}_{j}^{n}\right)\right]+\Delta t \mathbf{B}\left(\mathbf{V}_{j}^{n}\right), \\
\mathbf{V}_{j}^{n+1}=\frac{1}{2}\left(\mathbf{V}_{j}^{n}+\mathbf{V}_{j}^{*}\right)-\frac{\Delta t}{2 \Delta x}\left[\mathbf{F}\left(\mathbf{V}_{j}^{*}\right)-\mathbf{F}\left(\mathbf{V}_{j-1}^{*}\right)\right]+\frac{\Delta t}{2} \mathbf{B}\left(\mathbf{V}_{j}^{*}\right),
\end{gathered}
$$

where the notation $\mathbf{V}_{j}^{n} \equiv \mathbf{V}\left(x_{j}, t_{n}\right)$ is utilized with $\Delta x$ denoting the uniform grid spacing and $\Delta t$ is the time increment.

In the second step we focus on the derivative-dependent source terms disregarded in the first step and consider the system

$$
\begin{gathered}
\frac{\partial q}{\partial t}=s_{2} \delta^{2} W e \frac{\partial^{3} h}{\partial x^{3}}+\frac{q^{2}}{14 \Theta_{1}^{2} \Theta_{2}^{2}}\left(s_{3} \frac{\partial h}{\partial x}+s_{4} \frac{\partial u_{p}}{\partial x}\right)+ \\
\frac{q}{R e \Theta_{1} \Theta_{2}}\left(s_{5} \frac{\partial h}{\partial x}+s_{6} \frac{\partial u_{p}}{\partial x}\right)+\frac{\Theta_{1}}{R e \Theta_{2}}\left(s_{7} \frac{\partial h}{\partial x}+s_{8} \frac{\partial u_{p}}{\partial x}\right), \\
\frac{D a^{2} R e}{\phi} \frac{\partial u_{p}}{\partial t}=\delta^{2} \operatorname{ReWe} \frac{\partial^{3} h}{\partial x^{3}} .
\end{gathered}
$$

This system is to be solved over a time increment using the results from the first step as the initial conditions. We point out that $h$ remains constant for the second step. Therefore, the equations are decoupled, and furthermore, if equation (4.23) is solved first, then in both equations the $x$ derivatives 
are only applied to know quantities. So at a given spatial grid point $u_{p}$ is linear in time and equation (4.22) can be discretised in time by a simple second-order accurate predictor-corrector scheme.

The numerical method for solving the reduced model can be used to calculate the evolution of a perturbed equilibrium flow. Specifically, as the initial condition we take

$$
h=h_{s}+\epsilon \sin \left(\frac{2 \pi}{L} x\right), \quad q=1, \quad u_{p}=3,
$$

where $\epsilon$ is the amplitude of the imposed perturbation and $L$ is the length of the computational spatial domain to which we apply periodic boundary conditions. We can thus determine whether or not the solution tends to equilibrium and conclude the stability of perturbations with wavenumber $2 \pi / L$. We considered a range of small values of the initial amplitude, $10^{-10}<\epsilon<10^{-3}$. We are thus confident that our analysis applies to naturally occurring infinitesimal perturbations and is effective in determining the stability of the equilibrium flow.

By iterating over the flow parameters we can pinpoint under what conditions a particular perturbation mode is neutrally stable. We can thus generate the neutral stability curves and compare with those from the linear analysis. Such a comparison is presented in Figure 4.1. Here, for a given value of $K$ and thus $L=2 \pi / K$, we incremented the Reynolds number by small increases of 0.005 . For each $R e$ value we ran the simulation until the amplitude of the perturbation become steady. As Re was increased, at a particular point we found this amplitude to change from values less than $10^{-9}$ to values of the same order of magnitude as $10^{-2}$. We used this threshold as the condition for instability. As it can be seen in Figure 4.1, there is some disagreement between the nonlinear results and the prediction of the linear theory. More precisely, the band of moderately long modes that are linearly unstable for $R e<R e_{0}$ are predicted to be unstable only for $R e>R e_{0}$. As it is well know, linear instability only indicates that infinitesimal perturbations are amplified. However, as the perturbations grow, nonlinear terms become significant and can act to arrest the growth. The perturbation magnitudes necessary for the nonlinearity to take effect can still be very small, and if these levels are not exceeded the flow is practically stable. Our solutions with deviation from equilibrium of magnitude $10^{-9}$ could be caused by an initial growth that is then counteracted by nonlinearity. It must also be pointed out, however, that because the variations in these solutions are much smaller than the truncation error of our numerical method, we can not be entirely certain of their accuracy. Nevertheless, in either case we can conclude that the perturbation does not significantly alter the equilibrium solution for Reynolds numbers less than the threshold indicated above.

For flow parameter values which lead to instability of the equilibrium flow, the numerical calculation of the evolution can be continued until the growth of perturbations saturates and a secondary flow is established exhibiting permanent surface waves. In the impermeable substrate case the general structure of the waves is dictated by the degree of supercriticality of the flow conditions [28]. For supercritical conditions close to criticality the surface of the fluid film has a small-amplitude sinusoidal shape. For parameter values sufficiently beyond critical levels permanent solitary waves are generated on the surface of the fluid film. These waves have large heights and are preceded by high-frequency oscillations of significantly smaller amplitude. For flows down permeable inclines changes in the properties of the 


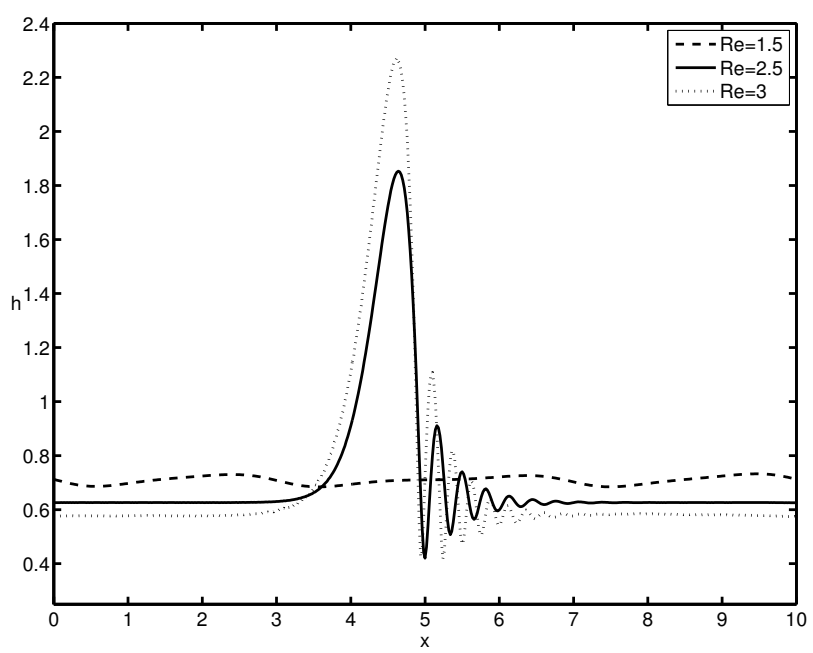

Figure 4.3: Permanent surface profiles for unstable flows with different Reynolds numbers and $D a=0.3$, $\cot \theta=1, \phi=0.01, \alpha_{B J}=1, K a=100$ and $d=1$.

substrate can affect the general form of the permanent surface profile, but this due to the direct impact on the critical conditions for the onset of instability. As it is illustrated in Figure 4.3, the progression of the types of waves with departure from criticality was found to be the same as the one described above for the basic impermeable bottom case. 


\section{Chapter 5}

\section{Conclusions}

In this thesis we studied the interfacial instability of a gravity-driven film flow with significant fluid filtration through the substrate. We considered an equilibrium flow that is steady and uniform in the streamwise direction. We determined the development of instability by implementing a model for the evolution of naturally occurring infinitesimal perturbations that interact with the equilibrium flow. Our goal was to examine the effect of various aspects of the porous substrate, such as its thickness, permeability and porosity, and how these factors combine with aspects of the film flow such as surface tension.

As part of our investigation, we carried out a linear stability analysis. As such, we linearized the governing equations with respect to perturbations imposed on the equilibrium solution. We obtained an eigenvalue problem with an Orr-Sommerfeld type equation, the solution of which allowed us to predict under what conditions different perturbations are amplified. As a result, we were able to determine the critical conditions at the threshold to flow instability, and the wavelength of perturbations that lead to instability at the onset. For the basic problem with an impermeable substrate, infinitely long perturbations (with zero wavenumber) generate the instability as the threshold is crossed. As it is well known, surface tension acts to suppress surface waves, but its effect diminishes as the wavelength is increased. Consequently, the critical condition for the flow to be unstable is not affected by surface tension and is expressed as $R e_{\text {crit }}=\frac{5}{6} \cot \theta$. This condition specifies the necessary level of inertia to cause instability for a given inclination. As the Reynolds number is increased ( and thus inertia intensified) beyond criticality, the range of unstable perturbation wavenumbers, $0 \leq K \leq K_{c}$, widens, i.e. $K_{c}$ increases with $R e$. Of course, for a fixed supercritical $R e$ value, $K_{c}$ decreases with surface tension.

In contrast to the basic problem, if the substrate is porous it is possible to have instability set in as a result of the amplification of a finite perturbation. This phenomenon occurs for sufficiently high permeability and sufficiently low porosity of the porous medium, and provided that the thickness of the substrate is sufficiently large. Furthermore, the surface tension of the fluid film must be sufficiently weak. At these levels, surface tension does in fact influence the onset of instability in the equilibrium flow, and as expected, it plays a stabilizing role.

Another important aspect that we examined is how the critical Reynolds number for the onset of 
instability is affected by the permeability of the substrate. Previous investigations [16] have considered small values for the permeability and the slip parameter imposed at the fluid-porous medium interface, and concluded that increasing the permeability destabilizes the flow. In our investigation we considered a wider range of parameter values, and found that increasing permeability can also have a stabilizing effect for certain parameter values.

We have also explored nonlinear effects on the stability of the flow. To facilitate a nonlinear analysis we simplified the governing equations by applying a long-wave approximation and a reduction in space dimensionality by implementing a weighted-residual method. In order to gauge the accuracy of the approximations a linear stability analysis was performed on the thus reduced model. The results proved to be in good agreement with those from the full equations. To carry out the nonlinear analysis, a numerical method was applied to the reduced model and used to calculate the evolution of sinusoidal perturbations of given wavelength and amplitude. The results, in contrast to those of the linear analysis, do not indicate that it is possible for a given flow to have instability of certain perturbations while longer ones are stable. This suggests that the perturbations with positive wavenumber that are found to be linearly unstable at the onset are in fact damped if nonlinear effects are not discarded, and the equilibrium flow is thus subject to nonlinear stability. 


\section{References}

1. Chakraborty, S. 2012 Dynamics and stability of a non-Newtonian falling film, Doctoral Dissertation, Université Paris Sud.

2. Gonputh, N. E. 2011 Film flow with temperture dependent fluid properties over heated inclined surfaces, MSc Dissertation, Ryerson University.

3. Sadiq, I. M. R., Usha, R. and Joo, S. 2010 Instabilities in a liquid film flow over an inclined heated porous substrate. Chem. Eng. Sci. 65, 4443.

4. Samanta, A., Goyeau, B. and Ruyer-Quil C. 2013 A falling film on a porous medium. J. Fluid Mech. 716, 414-444.

5. Kalliadasis, S., Ruyer-Quil, C., Scheid, B. and Velarde, M. 2012 Falling liquid films, Springer, London.

6. Benjamin, T. B. 1957 Wave formation in laminar flow down an inclined plane. J. Fluid Mech. 2, 554-574.

7. Yih, C.-S. 1963 Stability of liquid flow down an inclined plane. Phys. Fluids 6, 321-334.

8. Pascal, J. P. 1999 Linear stability of fluid flow down a porous inclined plane. J. Phys. D 32, 417-422.

9. Beavers, G. S. and Joseph, D. D. 1967 Boundary conditions at a naturally permeable wall. J. Fluid Mech. 30, 197-207.

10. Pascal, J. P. 2006 Instability of power-law fluid flow down a porous incline. J. Non-Newtonian Fluid Mech. 133, 109-120.

11. Sadiq, I. M. R. and Usha, R. 2008 Thin Newtonian film flow down a porous inclined plane: Stability analysis. Phys. Fluids 20, 022105.

12. Samanta, A., Ruyer-Quil, C., and Goyeau, B. 2011 A falling film down a slippery inclined plane. J. Fluid Mech. 684, 353-383. 
13. Pascal, J. P., DAlessio, S.J.D. 2010 Instabilities in gravity-driven flow over uneven permeable surfaces Int. J. Multiphase Flow 23, 449-459.

14. Ogden, K. A., DAlessio, S. J. D., and Pascal, J. P. 2011 Gravity-driven flow over heated, porous, wavy surfaces. Phys. Fluids 23, 122102.

15. Nield, D. A. and Bejan, A. 2006 Convection in porous media 3rd ed. Springer, New York.

16. Liu, R. and Liu, Q. S. 2009 Instabilities of a liquid film flowing down an inclined porous plane. Phys. Rev. E 80, 036316.

17. Thiele, U., Goyeau, B., Velarde, M. 2009 Stability analysis of thin film flow along a heated porous wall. Phys. Fluids 21, 014103.

18. Goyal, H., Kumar, A., Bandyopadhyay, D., Usha R. and Banerjee T. 2013 Instabilities of a confined two-layer flow on a porous medium: An Orr-Sommerfeld analysis. Chem. Eng. Sci. 97, 109-125.

19. Kumar, A., Goyal H., Banerjee, T., and Bandyopadhyay, D. 2013 Instability modes of a two-layer Newtonian plane Couette flow past a porous medium. Phys. Rev. E 87, 063003.

20. Jones, I. P. 1973 Low Reynolds number flow past a porous spherical shell. Proc. Camb. Phil. Soc. 73, 231-238.

21. Kandel, H. N., Pascal, J. P. 2013 Inclined fluid film flow with bottom filtration . Phys. Rev. E 88, 052405

22. Jackson, G. W. and James, D. F. 1986 The permeability of fibrous porous media. Can. J. Chem. Engng 64, 364-374.

23. Ruyer-Quil, C. and Manneville, P. 2000 Improved modeling of flows down inclined planes. Eur. Phys. J. B 15, 357-369.

24. Ruyer-Quil, C. and Manneville, P. 2002 Further accuracy and convergence results on the modeling of flows down inclined planes by weighted-residual approximations. Phys. Fluids 14, 170-183.

25. D'Alessio, S. J. D., Pascal, J. P. and Jasmine, H. A. 2009 Instability in gravity-driven flow over uneven surfaces. Phys. Fluids 21, 062105.

26. D’Alessio, S. J. D., Pascal, J. P., Jasmine, H. A. and Ogden K.A. 2010 Film flow over heated wavy inclined surfaces. J. Fluid Mech. 665, 418-456.

27. LeVeque, R. J. and Yee, H. C. 1990 A study of numerical methods for hyperbolic conservation laws with stiff source terms. J. Comput. Phys. 86, 187.

28. Chang, H.-C. 1994 Wave evolution on a falling film. Annu. Rev. Fluid Mech. 26, 103-136. 